\title{
Diversidad y patrones de distribución de coleópteros en la Región del Biobío, Chile: una aproximación preliminar para la conservación de la diversidad
}

\author{
Diversity and distributional patterns of beetles in the Biobío region, Chile: a preliminary \\ approach to the conservation of the diversity
}

OLIVIA E. VERGARA, VIVIANE JEREZ \& LUIS E. PARRA

\begin{abstract}
Departamento de Zoología, Facultad de Ciencias Naturales y Oceanográficas, Universidad de Concepción, Casilla 160-C, Concepción, Chile; e-mail para correspondencia: olvergar@udec.cl
\end{abstract}

\begin{abstract}
RESUMEN
Los coleópteros, por estar asociados con formaciones vegetales, presentar gran abundancia y diversidad ecológica, rangos de distribución restringidos y alto endemismo, constituyen buenos indicadores de la biodiversidad de un territorio. En la Región del Biobío, área crítica de conservación, estudios sobre la riqueza taxonómica y distribución de coleópteros son muy pocos a pesar de su carácter transicional y de constituir una zona de "puntos calientes" de diversidad a nivel mundial. Nuestros objetivos fueron conocer la composición taxonómica y representatividad regional de coleópteros, determinar sectores con mayor riqueza de especies y relacionar los patrones de distribución de las especies con formaciones vegetales y áreas silvestres protegidas de la región. A partir de un mapa dividido en cuadrículas y una base de datos de localidades de colecta obtenidos de literatura y colecciones de referencia, se confeccionó una matriz de presencia/ausencia de especies en la región. Para cada cuadrícula se estableció la riqueza de especies y, para determinar áreas de concentración de especies y su relación con las formaciones vegetales se realizó un análisis de parsimonia de endemismos (PAE). Se registraron 53 familias, 361 géneros y 664 especies de coleópteros, siendo Staphylinidae y Curculionidae las familias más diversas. Los lugares con mayor número de registros corresponden a Chillán, Concepción, y Cordillera de Nahuelbuta. El análisis de parsimonia agrupa las especies en tres sectores: (1) Sector Costero, (2) Cordillera de los Andes) y (3) Cordillera de Nahuelbuta. Estas áreas de concentración de especies no se relacionan con las áreas silvestres protegidas de la región.
\end{abstract}

Palabras clave: Insecta, Coleoptera, formaciones vegetacionales, Áreas Silvestres Protegidas del Estado, análisis de parsimonia de endemismos (PAE).

\begin{abstract}
Beetles constitute bioindicators because of their association with plant formations, their high abundance, ecological diversity, restricted geographic ranges and high endemism. The Biobío Region is a critical area for conserving the biodiversity because it represents a biotic transition zone and a hotspot of biodiversity, nevertheless the studies related with the taxonomic richness and distribution of beetles are very scarce. In this paper, we determined the taxonomic composition, the regional representation, and the species richness of beetles in this region. On the other hand, we determined areas that concentrate the majority of the species and the distribution patterns of insects related to plant formations and regional protected wild areas. A digitalized map of the region divided in quadrants and a database of distribution data of beetles were used to elaborate a matrix of presence/absence of species. The number of species contained in each quadrant was determined, and parsimony analysis of endemicity (PAE) was performed to determine areas concentrating the species richness, and its relation with plant formations. A total of 53 families, 361 genera and 664 species of beetles were found; Staphylinidae and Curculionidae were the most diverse families. Chillán, Concepcion and Nahuelbuta are localities with the highest species richness. Parsimony analysis of endemicity grouped species into three areas: (1) a coastal sector, (2) an Andean mountain range, and (3) the Nahuelbuta coastal range. These concentration areas of species are not related with regional protected wild areas.
\end{abstract}

Key words: Insecta, Coleoptera, plant formations, State Wild Protected Areas, parsimony analysis of endemicity (PAE). 


\section{INTRODUCCIÓN}

La región del Biobío, zona de transición entre los componentes de la flora esclerófila de Chile central y del bosque templado lluvioso propio del dominio subantártico (Teneb et al. 2004) presenta como particularidades biológicas una notable riqueza vegetacional (Cisternas et al. 1999), un área máxima de concentración de distintos taxa (Villagrán \& Hinojosa 1997a, 1997b, Teneb et al. 2004) y altos niveles de endemismo (Arroyo 2002). Sin embargo, en esta región se encuentra el $13 \%$ de la población humana del país (INE 2002), lo que ha generado en los últimos 50 años una gran alteración de sus ecosistemas, reflejado principalmente en la fragmentación de los bosques costeros de tipo esclerófilo y caducifolio (Briones \& Jerez 2004). A pesar de ello, y aunque existe prioridad regional en considerarla como un área crítica de conservación (Elgueta 1995, Arroyo 2002), solo el 2,8 \% de la superficie se encuentra protegida, territorio que alcanza hasta la fecha cinco áreas silvestres protegidas por el Estado (sensu CONAF-CONAMA-BIRP 1999, IbarraVidal et al. 2005).

Para cuantificar la riqueza de especies y su representatividad en las comunidades, se utilizan básicamente inventarios florísticos y/o faunísticos reunidos en bases de datos, asociado con sistemas de información geográfica (SIG) (Veloso \& Núñez 1998, Elgueta 2000a, Jerez 2000a, Funk et al. 2002, Pizarro-Araya \& Jerez 2004). Otros métodos como el PAE (análisis de parsimonia de endemismos), utilizados por la biogeografía histórica y ecológica, permiten analizar la distribución espacial de las especies (Morrone \& Ruggiero 2001, Morrone 2004) y determinar áreas que concentren la mayor riqueza específica y a la vez sean únicas en cuanto a su composición biótica (Jerez 2000a, Cavieres et al. 2001, Cavieres et al. 2002, Roig-Juñent et al. 2002, Escalante \& Morrone 2003, RoigJuñent \& Debandi 2004, Rovito et al. 2004).

Los insectos y específicamente los coleópteros, por su gran abundancia y diversidad ecológica, constituyen buenos indicadores de la biodiversidad de un territorio (Morrone \& Ruggiero 2001, Yeates et al. 2002) y por estar asociados con las formaciones vegetales donde actúan como depredadores, herbívoros, polinizadores y/o descomponedores de materia orgánica, presentan rangos de distribución restringidos (Solervicens 1995, Jerez 2000a). Sin embargo, estudios sobre la distribución de coleópteros en Chile y particularmente en la Región del Biobío, son muy pocos, de modo que en términos de conservación, estos insectos deberían ser clasificados como inadecuadamente conocidos (Elgueta 2000a, Arroyo 2002).

En Chile, los coleópteros están representados por 96 familias, 1.196 géneros y 3.947 especies, que corresponden a un $30 \%$ del total de especies de insectos descritas para el país (Elgueta 2000a). La mayoría de los géneros están poco diversificados y presentan altos grados de endemismo (Solervicens 1995, Grez et al. 2002). Dado que la región del Biobío es una zona de transición florística y faunística y parte del "punto caliente" de biodiversidad de Chile Central con prioridades de conservación (Arroyo 1999, Myers et al. 2000, Rovito et al. 2004) y a pesar del gran deterioro de sus comunidades, debería albergar aún, una gran diversidad de especies de coleópteros en relación con la diversidad descrita para el país. Por otra parte y dado que estos insectos están asociados a la vegetación, cabría esperar que los coleópteros mostraran áreas de distribución comunes para grupos de especies, concordantes a su vez con las formaciones vegetales descritas para este sector.

Los objetivos del presente trabajo son: (1) conocer la composición taxonómica de los coleópteros para la Región del Biobío, (2) determinar patrones de distribución de las especies y (3) reconocer sectores que presenten la mayor riqueza. Finalmente, se espera determinar si existe concordancia entre estos últimos sectores con las formaciones vegetales y con las áreas silvestres protegidas por el Estado en la Región del Biobío.

\section{MATERIALES Y MÉTODOS}

Composición taxonómica y diversidad de coleópteros de la Región del Biobío

Se elaboró una base de datos de distribución a partir de registros de localidades a partir de literatura especializada, informes técnicos y 
colecciones científicas entre los años 1953 y 2004, así como de prospecciones en terreno. Se revisaron colecciones entomológicas de referencia del Museo Nacional de Historia Natural (MNHN), Chile; Museo de Zoología (UCCC), Universidad de Concepción, Chile, e Instituto de Entomología de la Universidad Metropolitana de Ciencias de la Educación (UMCE), Chile. La base de datos incluye para cada especie un número identificador (ID), localidad de recolección y su ubicación geográfica (latitud-longitud) (López \& Gutiérrez 1983). Para la ordenación sistemática de los taxa se siguió a Lawrence \& Newton (1995) (sensu Arias 2000) y no se consideraron las subespecies, ni especies con referencia dudosa.

Para la ubicación de puntos de registros, se utilizó un mapa regional digitalizado, escala 1:500.000 y una grilla de 86 cuadrículas de $0.25 \times 0.25$ grados de latitud y longitud (cada una equivalente a una superficie aproximada a $692 \mathrm{~km}^{2}$ ); diecinueve cuadrículas que representan menos de la mitad del territorio en estudio se fusionaron con la cuadrícula adyacente obteniendo finalmente un total de 67 cuadrículas (Roig-Juñent et al. 2002, Teneb et al. 2004). El territorio insular de la región está representado en las cuadrículas 14 (Isla Quiriquina), 82 (Isla Mocha) y 23-33 (Santa María) respectivamente (Fig. 1).

\section{Análisis de extrapolación GAP}

Para predecir la presencia potencial de especies en cuadrículas donde no han sido detectadas y para optimizar el análisis de parsimonia de endemismos (PAE), se efectuó un análisis GAP ("Gap Analysis Program") superponiendo diferentes capas de información según la metodología señalada por Scott \& Jennings (1998) y Pliscoff (2003). Estas capas incluyen la base de datos con los registros de colecta de las especies de coleópteros, un mapa digital con la distribución de las formaciones vegetales descritas por Gajardo (1994) y las curvas de relieve de la región (Diepenbroek et al. 2000), con lo cual se extrapoló la distribución de las especies asociadas con similar formación vegetal y altitud.

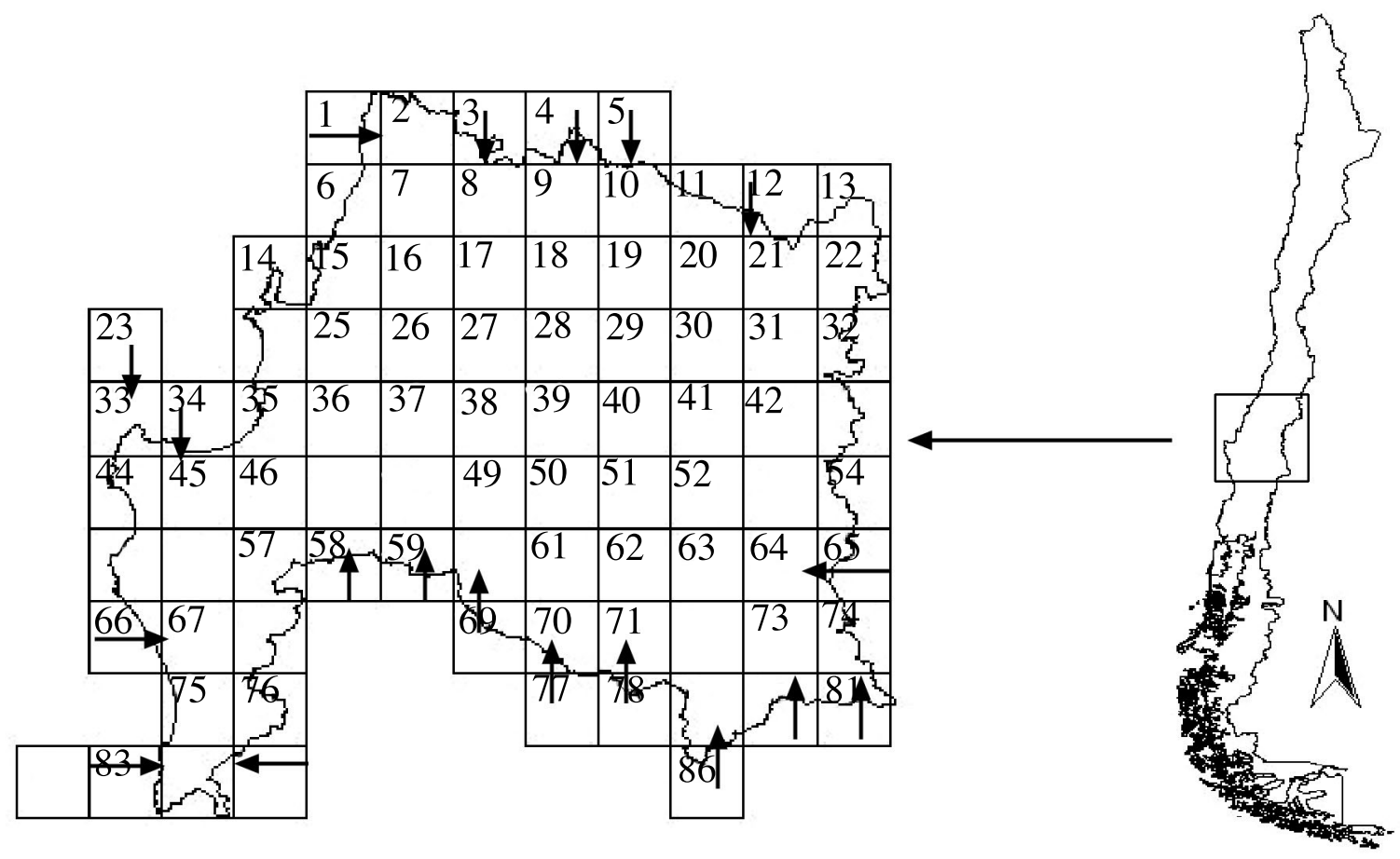

Fig. 1: Mapa de la Región del Biobío mostrando la grilla de 0,25 x 0,25 grados de latitud y longitud y la unión de cuadrículas.

Map of the Biobío Region showing the grid of $0.25 \times 0.25$ degree latitude and longitude and the union of quadrants. 


\section{Determinación de áreas de concentración de especies}

Para el PAE se confeccionó una matriz de presencia/ausencia (valores 1 o 0 ) de 292 especies que presentaron más de 1 registro de colecta en 50 cuadrículas. Esta selección se debe a que los taxa que se encuentran en una única cuadrícula son equivalentes a autapomorfías por lo cual no son útiles para establecer relaciones entre cuadrículas (Fattorini 2002, Roig-Juñent et al. 2002).

Para el procesamiento de la matriz se siguió la metodología propuesta por Morrone (1994), que permite agrupar áreas por taxa compartidos, análogos a los caracteres de acuerdo con la solución más parsimoniosa (Posadas \& MirandaEsquivel 1999, Pizarro-Araya \& Jerez 2004, Rovito et al. 2004). Para enraizar el o los cladogramas resultantes, se agregó una cuadrícula hipotética con ausencias (0) (Cavieres et al. 2002). La matriz fue analizada utilizando un algoritmo de simplicidad y mediante una búsqueda heurística (hold 1000, hold d/10 y multi*100) con el programa NONA 2.0 (Goloboff 1993) y ejecutado a través del programa Winclada (Nixon 2000) se obtuvo un árbol de consenso estricto. Para obtener el nivel de soporte de las áreas de concentración de especies se calculó el índice de "bootstrap", tomando en cuenta sólo aquellos grupos de cuadrículas que formaron un clado y cuya unión estuvo sustentada por la presencia de dos o más taxa (Morrone 1994, Escalante \& Morrone 2003, Pizarro-Araya \& Jerez 2004).

Posteriormente y para detectar áreas superpuestas, se aplicó el procedimiento PAEPCE (PAE con eliminación progresiva de caracteres) desarrollado por García-Barros (2003) para ser aplicada en árboles de consenso de baja resolución; esto requiere sucesivos análisis de parsimonia desactivando las especies sinapomórficas que sirvieron para identificar un área de concentración de especies y favorece la emergencia de agrupaciones de áreas alternativas a la más parsimoniosa.

Relación entre áreas de concentración de especies, formaciones vegetales y áreas silvestres protegidas

Para determinar si existe correspondencia entre los patrones de distribución de los taxa y las formaciones vegetales descritas para la región, se relacionaron los grupos obtenidos anteriormente con el PAE-PCE, con un mapa digital de las formaciones vegetales (Gajardo 1994) y áreas silvestres protegidas por el Estado propuestas para la región (Comisión Nacional del Medio Ambiente, Biobío 2003) utilizando el programa Arc View 3.2 (ESRI 1996).

\section{RESULTADOS}

Composición taxonómica y diversidad de coleópteros de la región del Biobío

En la Tabla 1 se muestra la diversidad de coleópteros descrita para la región del Bíobío, la que está representada por 53 familias, 361 géneros y 664 especies, valores que representan el $55 \%$ de familias, $30 \%$ de géneros y $17 \%$ de especies en relación con la diversidad de coleópteros descritos a nivel nacional (Elgueta 2000a). Se muestra además, para cada familia, la riqueza de géneros y especies y su porcentaje de representatividad a nivel regional. Las familias más diversificadas en géneros y especies son Staphylinidae y Curculionidae seguidas por Cerambycidae, Chrysomelidae y Tenebrionidae. A nivel de géneros, Eurymetopum, Sciacharis, Conognatha y Mordella presentaron el mayor número de especies (Tabla 2).

En cuanto a localidades de colecta (Fig. 2), la mayoría de los puntos de registros están concentrados en sectores preandinos (Cordillera de Chillán, 36 34' S, 72 $06^{\prime}$ O), sector costero (Talcahuano, Concepción y alrededores, 36² $43^{\text {' }}$ S, $73^{\circ} 07^{\prime}$ O) y parte de la Cordillera de la Costa (Cordillera de Nahuelbuta, $37^{\circ} 46^{\prime}$ S, $73^{\circ} 02^{\prime}$ O). En la Fig. 3 se observa que la cuadrícula 24 es la que presenta la mayor riqueza con 258 especies, seguidas por las cuadrículas 31 (170 especies), 68 (134 especies), 30 (112 especies), 18 (102 especies) y 14 (99 especies). Las cuadrículas con menor riqueza de especies corresponden a la 4 , $12,23,27,33,51,58,75$. Finalmente 18 cuadrículas ubicadas principalmente en el límite norte, este, sur-este y sur de la región no presentan ningún registro de especies. En cuanto al patrón de distribución de las familias más representativas, Staphylinidae (Fig. 4A), Curculionidae (Fig. 4B), Chrysomelidae (Fig. 4C) y Tenebrionidae (Fig. 4D) se concentran principalmente en el sector costero de la 
provincia de Concepción y cordillera pre-andina de Chillán. Apterodorcus bacchus, Aegorhinus superciliosus, Astylus trifasciatus, Ceroglossus chilensis, Oligocara nitidum y Procalus mutans presentan el mayor número de puntos de registros de localidades.

\section{TABLA 1}

Diversidad de familias, géneros y especies de coleópteros y porcentaje total de géneros y especies para la región del Biobío (\% R)

Diversity of families, genera and species of beetles and total percentage of genera and species for the Biobío region (\%R)

\begin{tabular}{|c|c|c|c|c|c|}
\hline & Familia & Número de géneros & Porcentaje (\% R) & Número de especies & Porcentaje (\% R) \\
\hline 1 & Anobiidae & 7 & 1,9 & 7 & 1,05 \\
\hline 2 & Anthicidae & 1 & 0,27 & 3 & 0,45 \\
\hline 3 & Anthribidae & 2 & 0,55 & 2 & 0,30 \\
\hline 4 & Attelabidae & 1 & 0,27 & 2 & 0,30 \\
\hline 5 & Belidae & 2 & 0,55 & 2 & 0,30 \\
\hline 6 & Biphyllidae & 1 & 0,27 & 1 & 0,15 \\
\hline 7 & Bostrichidae & 4 & 1,10 & 4 & 0,60 \\
\hline 8 & Brentidae & 1 & 0,27 & 2 & 0,30 \\
\hline 9 & Bruchidae & 3 & 0,83 & 3 & 0,48 \\
\hline 10 & Buprestidae & 16 & 4,43 & 35 & 5,27 \\
\hline 11 & Cantharidae & 3 & 0,83 & 6 & 0,90 \\
\hline 12 & Carabidae & 27 & 7,48 & 43 & 6,48 \\
\hline 13 & Cerambycidae & 30 & 8,31 & 46 & 6,93 \\
\hline 14 & Ceratocanthidae & 2 & 0,55 & 3 & 0,45 \\
\hline 15 & Chrysomelidae & 27 & 7,48 & 47 & 6,92 \\
\hline 16 & Cleridae & 11 & 3,04 & 32 & 4,82 \\
\hline 17 & Coccinellidae & 12 & 3,32 & 15 & 2,26 \\
\hline 18 & Cryptophagidae & 1 & 0,27 & 1 & 0,15 \\
\hline 19 & Cucujidae & 2 & 0,55 & 2 & 0,30 \\
\hline 20 & Cupedidae & 1 & 0,27 & 1 & 0,15 \\
\hline 21 & Curculionidae & 49 & 13,57 & 83 & 12,5 \\
\hline 22 & Dermestidae & 1 & 0,27 & 1 & 0,15 \\
\hline 23 & Dytiscidae & 3 & 0,83 & 7 & 1,05 \\
\hline 24 & Elateridae & 12 & 3,32 & 21 & 3,16 \\
\hline 25 & Elmidae & 1 & 0,27 & 1 & 0,15 \\
\hline 26 & Erotylidae & 2 & 0,55 & 7 & 1,05 \\
\hline 27 & Eucnemidae & 1 & 0,27 & 1 & 0,15 \\
\hline 28 & Geotrupidae & 2 & 0,55 & 4 & 0,60 \\
\hline 29 & Gyrinidae & 1 & 0,27 & 1 & 0,15 \\
\hline 30 & Haliplidae & 1 & 0,27 & 1 & 0,15 \\
\hline 31 & Histeridae & 3 & 0,83 & 4 & 0,60 \\
\hline 32 & Hydrophilidae & 5 & 1,39 & 7 & 1,05 \\
\hline 33 & Lampyridae & 2 & 0,55 & 5 & 0,75 \\
\hline 34 & Lathridiidae & 2 & 0,55 & 2 & 0,30 \\
\hline 35 & Leiodidae & 13 & 3,60 & 19 & 2,86 \\
\hline 36 & Lucanidae & 5 & 1,39 & 12 & 1,81 \\
\hline 37 & Meloidae & 1 & 0,27 & 1 & 0,15 \\
\hline 38 & Melyridae & 2 & 0,55 & 5 & 0,75 \\
\hline 39 & Mordellidae & 1 & 0,27 & 13 & 1,96 \\
\hline 40 & Nitidulidae & 1 & 0,27 & 1 & 0,15 \\
\hline 41 & Phengodidae & 1 & 0,27 & 2 & 0,30 \\
\hline 42 & Protocucujidae & 1 & 0,27 & 3 & 0,45 \\
\hline 43 & Salpingidae & 1 & 0,27 & 1 & 0,15 \\
\hline 44 & Scarabaeidae & 15 & 4,16 & 29 & 4,37 \\
\hline 45 & Scraptiidae & 1 & 0,27 & 1 & 0,15 \\
\hline 46 & Scydmaenidae & 1 & 0,27 & 15 & 2,26 \\
\hline 47 & Silphidae & 1 & 0,27 & 1 & 0,15 \\
\hline 48 & Sphindidae & 1 & 0,27 & 2 & 0,30 \\
\hline 49 & Staphylinidae & 50 & 13,85 & 101 & 15,21 \\
\hline 50 & Tenebrionidae & 22 & 6,09 & 51 & 7,68 \\
\hline 51 & Tetratomidae & 1 & 0,27 & 1 & 0,15 \\
\hline 52 & Trogidae & 2 & 0,55 & 2 & 0,30 \\
\hline 53 & Zopheridae & 2 & 0,55 & 2 & 0,30 \\
\hline \multicolumn{2}{|c|}{ Total } & 53 & 361 & & 664 \\
\hline
\end{tabular}


TABLA 2

Listado de especies de coleópteros en la Región del Biobío y su ubicación en las cuadrículas para análisis de parsimonia de endemismos. En paréntesis se muestran las cuadrículas extrapoladas en el análisis GAP; UCCC = Museo de Zoología de la Universidad de Concepción (Concepción, Chile), UMCE = Instituto de Entomología de la Universidad Metropolitana de Ciencias de la Educación

(Santiago, Chile), MNHN = Museo Nacional de Historia Natural (Santiago, Chile)

Beetle listing in the Biobío region according to their location within quadrants for the parsimony analysis. Quadrant extrapolations during the GAP analysis are in parentheses; UCCC = Museo de Zoología of Universidad de Concepción (Concepción, Chile), UMCE = Instituto de Entomología de la Universidad Metropolitana de Ciencias de la Educación

(Santiago, Chile), MNHN = Museo Nacional de Historia Natural (Santiago, Chile)

ID Taxa

28 Anthaxia auronotata Bily

29 Anthaxia concinna (Mannh.)

30 Anthaxia cupriceps F. \& G.

32 Buprestis novemmaculata L.

33 Chrysobothris bothrideres (F. \& G.)

34 Conognatha azarae (Phil. \& Phil.)

35 Conognatha azurea Phil.

36

37

Cicindela chilensis Audovin \& Brullé

Conognatha chilensis (Guér.)

Conognatha costipennis (G.)

Conognatha errata (F. \& G.)

Conognatha germaini (Théry)

Conognatha humeralis (Phil.)

Conognatha souverbii (G.)

Conognatha viridiventris (Sol.)

Cylindrophora verecunda Er.

Dactylozodes rousseli (Sol.)

Dactylozodes semivittata (F. \& G.)

Ectinogonia buqueti (Spin.)

Epistomentis pictus (Gory)

Hypoprasis elegans (Phil. \& Phil.)

Mendizabalia germaini (Kerr.)

Philandia valdiviana (Phil. \& Phil.)

Polycesta costata Sol.

Pterobothris corrosus F. \& G.

Trigonogenium angulosum (Sol.)

Tyndaris marginella F. \& G.

Tyndaris planata (Lap. \&Gory)

\section{Carabidae}

Abropus carnifex Fabr.

Calosoma vagans Dej.

Ceroglossus chilensis Esch.

\section{Cicindela nahuelbutae (Peña)}

Cnemalobus germaini Putzeys

Cyanotarus andinus $\mathrm{G}$.

Dromius maculipennis (Sol.)

Euproctinus fasciatus (Sol.)
Conognatha chabybaeofasciata G. \& Kerr. 18, 19, 30 (20)

Callidula nigrofasciata (Sol.)

\section{Cuadrículas (en paréntesis, Fuente \\ extrapolación GAP)}

\section{$31,68(57)$}

$2,7,8,14,18,19,24,26,30$,

$31,35,48,68,76(25)$

$18,30,53$

$14,17,18,24,36,37,48,49$,

$52,60(25)$

$15,18,30,31,53$

$10,18,19(11)$

30,31

$2,24,76$

$18,30,31$

$8,10,18,19,24,30,31,57$, $60,68,76(9)$

$31,53,57,60,68(58,42)$

$30,56,68(57)$

$8,30,31$

$18,19,24,31,45,67(14,35)$

$8,18,19,24,25,30,31,60$ (9)

$11,30,31,37,53$

30,31

$2,25,30,31,35,53(24,42)$

$2,8,18,19,21,30,31,56,67$, $68,76(57)$

$30,31,45,53,68$

31,72

$2,31,45,56,68(57)$

$17,19,24,25,30,68$ (18)

$18,31,68$

$30,31,53(42)$

18,25

$30,31,53$

\footnotetext{
$31,67,68(57,56,30)$

$20,24,35,41(25,14,30)$

$2,14,24,26,30,35,45,53$

$(25,20,42)$

$2,8,14,17,18,20,24,25,26$,

$31,35,36,39,41,47,48,49$,

$57,68,72,76,82(27,30,37$,

$38,58)$

$17,30,35,36,57,68$

$(46,58,24)$

$48,56,68(57)$

$18,20,30,31,61(19,62)$

$7,20,41(2,6,30)$

36,45 (37)

$25,26,36(37)$
}

UCCC, Moore (1985)

UCCC, UMCE, MNHN

UCCC, MNHN

UCCC, MNHN

UCCC, Moore (1987)

UCCC, Moore (1981)

UCCC, MNHN

Moore (1981)

UCCC, MNHN, Moore (1981)

UCCC, Moore (1981)

UCCC, Moore (1981)

UCCC, Moore (1981)

UCCC, Moore (1981)

UCCC, Moore $(1981,1986)$

UCCC, MNHN, Moore $(1981,1986)$

UCCC, MNHN

UCCC, MNHN, UMCE

UCCC

UCCC, MNHN

UCCC, MNHN

Moore (1987)

Bellamy \& Moore (1990)

UCCC, Moore (1985)

UCCC

Moore (1985)

UCCC, MNHN

$\mathrm{UCCC}$

UCCC, Moore (1990)

UCCC

UCCC

UCCC

UCCC, MNHN, Mendoza \& Jerez (2001)

UCCC, MNHN

UCCC

Roig-Juñent (1994)

UCCC

UMCE

UMCE 
TABLA 2 (continuación)

\begin{tabular}{|c|c|c|c|}
\hline ID & Taxa & $\begin{array}{l}\text { Cuadrículas (en paréntesis, } \\
\text { extrapolación GAP) }\end{array}$ & Fuente \\
\hline 102 & Pachymorphus aerea (Dej.) & $20,24,36,41(30)$ & UCCC \\
\hline 103 & Laemostenus complanatus (Dej.) & $14,18,24(35,25)$ & UCCC \\
\hline 104 & Lebia azurea Sol. & $2,20,26$ & UCCC, UMCE \\
\hline 107 & Mimodromius cyanipennis Brullé & $2,24,41$ & UCCC \\
\hline 109 & Monolobus testaceus Sol. & 2,24 & UCCC \\
\hline 111 & Megachile pollinosa Spin. & $2,14,17,24,29,76$ & UCCC, Raw (2004) \\
\hline 81 & Nothocys marginatus (Sol.) & 31,82 & Jeannel (1962b) \\
\hline 87 & Notholopha stricticolle (G.) & 30,72 & Jeannel (1962b) \\
\hline 113 & Trirammatus aereus (Dej.) & $\begin{array}{l}14,20,24,26,29,41,49,57 \\
76,82(25)\end{array}$ & UCCC \\
\hline 114 & Trirammatus unistriata (Dej.) & $\begin{array}{l}8,14,20,26,31,35,39,41 \\
49,52,67,82(24)\end{array}$ & $\mathrm{UCCC}$ \\
\hline & 3. Cerambycidae & & \\
\hline 118 & Acanthinodera cummingii (Hope) & $14,20,24,26,41,53,60(25)$ & UCCC \\
\hline 119 & Aconopterus cristatipennis $\mathrm{B} 1$. & $2,14,24,29,35,60(45)$ & UCCC \\
\hline 120 & Adalbus crassicornis F. \& G. & $18,31,37$ & UCCC \\
\hline 122 & Callisphyris apicicornis F. \& G. & $14,18,24,31,35,39(45)$ & UCCC \\
\hline 124 & Callisphyris macropus New. & $14,15,24,35,39,57(25)$ & UCCC \\
\hline 126 & Calydon submetallicum (B1.) & $18,24,30,31,57(20)$ & UCCC \\
\hline 127 & Cheloderus childreni Gray & 30,68 & UCCC \\
\hline 128 & Chenoderus bicolor F. \& G. & 18,72 & UCCC \\
\hline 129 & Chenoderus testaceus (B1.) & $14,24,35,36,45,76(25)$ & UCCC, UMCE, Artigas (1969-1970) \\
\hline 130 & Chenoderus tricolor F. \& G. & $8,53,76$ & $\mathrm{UCCC}$ \\
\hline 131 & Colobura alboplagiata B1. & 26,76 & UCCC \\
\hline 133 & Grammicosum flavofasciatum (B1.) & $18,24,31$ & UCCC \\
\hline 134 & Hebestola humeralis $\mathrm{B} 1$. & $2,14,24,60$ & UCCC \\
\hline 135 & Hebestola vitticollis $\mathrm{B} 1$. & $2,45,67(35)$ & UCCC \\
\hline 138 & Holopterus annulicornis Phil. & 24,30 & UCCC, Fichet \& Barriga (1990) \\
\hline 146 & Microcleptes araneus New. & $2,14,24$ & UCCC \\
\hline 152 & Phoracantha semipunctata (Fabr.) & $18,24,30$ & UCCC \\
\hline 153 & Phymatioderus bizonatus (B1.) & $14,18,24,57,60$ & UCCC \\
\hline 154 & Planopus laniniensis Bos. & 30,31 & MNHN \\
\hline 155 & Platynocera rubriceps $\mathrm{B} 1$. & $24,45,76(35)$ & UCCC, UMCE \\
\hline 157 & Sybilla coemeterii (Thomson) & 31,68 & UCCC, Cerda (1973) \\
\hline 160 & Sybilla krahmeri Cerda & $30,31,68$ & MNHN, Cerda (1973) \\
\hline 161 & Sybilla livida $\mathrm{G}$ & 30,31 & UCCC, Cerda (1973) \\
\hline & 4. Chrysomelidae & & \\
\hline 168 & Ambrotodes elegans (B1.) & $31,68,76$ & UCCC \\
\hline 171 & Chelymorpha varians $\mathrm{B} 1$ & $\begin{array}{l}7,14,20,24,26,29,35,36, \\
39,57(25)\end{array}$ & $\mathrm{UCCC}$ \\
\hline 172 & Chlamisus apricarius (Lac.) & $17,26,31(27)$ & MNHN \\
\hline 173 & Chrysolina gemellata (Rossi) & $8,16,20,31,53,60(30)$ & UCCC, MNHN \\
\hline 175 & Dachrys succincta $($ Er.) & $\begin{array}{l}7,16,21,24,30,39,57,67 \\
68(58)\end{array}$ & UCCC \\
\hline 176 & Dictyneis asperatus (B1.) & $\begin{array}{l}2,14,15,16,17,20,24,29, \\
35,36,45(19,18,45)\end{array}$ & UCCC, Jerez (1991) \\
\hline 178 & Dictyneis conspurcatus (B1.) & $2,14,15,24,45(35,45)$ & Jerez (1991) \\
\hline 179 & Dictyneis terrosus (Phil. \& Phil.) & $14,25,76$ & Jerez $(1991)$ \\
\hline 180 & Hornius grandis (Phil. \& Phil.) & $16,24,26,72(25)$ & UCCC, Jerez \& Ibarra-Vidal (1992) \\
\hline 181 & Jansonius aeneus $\mathrm{B} 1$. & $10,20(11)$ & UCCC, MNHN, Askevold \& Lesage (1990) \\
\hline 183 & Kuschelina decorata (B1.) & $6,30,68$ & UCCC, MNHN \\
\hline 184 & Lexiphanes variabilis (B1.) & $26,38(37)$ & MNHN \\
\hline 185 & Lysathia virescens (Phil. \& Phil.) & $14,45,82$ & MNHN \\
\hline 186 & Lysathia atrocyanea (Phil \& Phil) & $16,21,24,56,67,68(57)$ & $\begin{array}{l}\text { UCCC, MNHN, UMCE, Jerez } \\
(1988,2000 b)\end{array}$ \\
\hline 187 & Macrohaltica janthina (B1.) & $17,24,26(25)$ & MNHN \\
\hline 188 & Mastacanthus suturalis (Suffr.) & 24,56 & UCCC \\
\hline 189 & Mylassa chachalloi Monros. & $30,31,68$ & UCCC \\
\hline 190 & Mylassa crassicollis (B1.) & $2,8,14,24,29,30,56,68(57)$ & UCCC, MNHN \\
\hline 191 & Mylassa discariana Monros. & $24,25,30,68$ & UCCC \\
\hline 192 & Mylassa obliquata Monros. & $31,36,68$ & UCCC \\
\hline
\end{tabular}


TABLA 2 (continuación)

\begin{tabular}{ll}
\hline ID & Taxa \\
\hline 193 & Mylassa pectinicornis (Suffr.) \\
196 & Pachybrachis gayi (B1.) \\
198 & Pachybrachis signatipennis (B1.) \\
199 & Pataya nitida (Phil. \& Phil.) \\
200 & Phaedon cyanopterum Guér. \\
202 & Procalus mutans (B1.)
\end{tabular}

203 Procalus reduplicatus Bech.

204 Procalus silvai Jerez

209 Psathyrocerus fulvipes B1.

210 Psathyrocerus oblongus B1.

213 Stenomela pallida Er.

\section{Cleridae}

215 Calendyma chiliensis (Lap.)

217 Eurymetomorphon biguttatus Solerv.

218 Eurymetomorphon inaequalicolle Pic

219 Eurymetopum brevevittatum (Pic)

220 Eurymetopum eburneocinctum (Spin.)

221 Eurymetopum fridigum (Chev.)

223 Eurymetopum iridescens (Pic)

224 Eurymetopum longulum (Spin.)

225 Eurymetopum maculatum B1.

226 Eurymetopum modestum (Phil. \& Phil.)

227 Eurymetopum obscurum (Phil. \& Phil.)

228 Eurymetopum parallelum (F. \& G.)

229 Eurymetopum prasinum (Spin.)

230 Eurymetopum rudibum (Chevr.)

231 Eurymetopum semiprasinum (Chevr.)

232 Eurymetopum semirufum (F. \& G.)

233 Eurymetopum viride (Phil. \& Phil.)

235 Hylodanacea impressa(Sol.)

237 Natalis impressus (Spin.)

240 Necrobia rufipes De Geer

242 Notocymatodera dimidiata (G.)

243 Silviella acutipenne (Spin.)

244 Silviella nudatum (Spin.)

245 Silviella pehuen Solerv.

246 Solervicensia ovatus (Spin.)

\section{Coccinelidae}

247 Adalia angulifera (Muls.)

248 Adalia bipunctata (L.)

249 Adalia deficiens Muls.

252 Coleomegilla quadrifasciata (Shoen.)

254 Eriopis connexa $\mathrm{G}$.

255 Hyperaspis germaini Crotch.

259 Psyllobora picta G.

261 Scymnus loewii Muls.

\section{Curculionidae}

269 Acalles litturatus B1.

270 Acalles moestus B1.

273 Aegorhinus albolineatus (B1.)

275 Aegorhinus maulinus Elgueta

277 Aegorhinus nodipennis (Hope)

278 Aegorhinus phaleratus (Er.)

Cuadrículas (en paréntesis, Fuente

extrapolación GAP)

$\begin{array}{ll}31,68 & \text { UCCC } \\ 24,26,30,31,56,68(25) & \text { MNHN } \\ 2,29,56,68(57) & \text { MNHN } \\ 14,24(35) & \text { UCCC } \\ 19,21,24,26,30,48(25) & \text { UCCC }\end{array}$

$2,7,14,15,17,20,24,26$, $29,31,37,45,48,53,68$

$(6,16,25,35)$

UCCC, Jerez (1992), Cortés et al. (1993), Jerez \& Centella (1996)

$7,14,16,17,24,26,29,31,35$, 37, 39, 57, $68(15,25,36,38) \quad$ UCCC, Jerez (1992), Cortés et al. (1993) 17,56

$31,52,53,64,72,74,76$

$52,64,72,76(63)$

$\mathrm{UCCC}$

$\mathrm{UCCC}$

UCCC

$14,24,35,36(45) \quad$ Jerez (1995)

$8,30,31$

$18,33,45,72(45)$

$31,56,68$

$31,68,72$

$24,31,76$

$31,56,67,68,72(57)$

$31,53,56,68,72(42)$

$2,17,24,26,29,68(25)$

2, 68

$18,21,24,30,31,53,64,68$ (20)

31,68

$31,56,68(57)$

$2,24,38$

31,72

$16,25,31,52,72$

$24,29,31,56,68(30,57,67)$

$31,45,56,68,72$

$26,30,72$

14,24

18,24

$2,10,24,31$

10,56

$2,18,24,25,31,56,68,72,82$

$56,68,72(57)$

$2,17,26,31,36,67$

$17,25,26$

$2,7,14,17,19,21,24(18)$

$7,14,17,24,25,26(15,16)$

21,24

$15,21,34$

21,25

2,24

$17,26(27,16)$

$24,45,82(35)$

14,25 (24)

$7,24,30$

$7,18,24,30,36,45$ (35)

$2,7,14,15,24,26,30,35$,

$56,68(16,25)$

24,35
UCCC, Solervicens (2002)

UCCC, Solervicens (1991)

Solervicens (1991)

Solervicens (1986)

Solervicens (1986)

Solervicens (1986)

Solervicens (1986)

MNHN, Solervicens (1986)

Solervicens $(1986,2002)$

Solervicens (1986)

Solervicens (1986)

Solervicens (1986)

UCCC, Solervicens (1986)

Solervicens (1986)

UCCC, Solervicens (1986, 2002),

Solervicens (1986)

Solervicens (1986)

MNHN

UCCC

UCCC, MNHN

UCCC, Solervicens (1996)

Solervicens (1987)

Solervicens (1987), UCCC

Solervicens $(1987,2002)$

Solervicens (2001)

UCCC, MNHN, UMCE

UCCC, MNHN

UCCC, MNHN

UCCC, MNHN

UCCC, Solervicens \& Estrada (2002),

Camus \& Barahona (2002)

UMCE, MNHN

UCCC

MNHN, UMCE

MNHN, Elgueta \& Cekalovic (1999)

UCCC

UCCC, Kuschel (1957), Elgueta (2000b)

MNHN, UMCE, Elgueta (2000b)

UCCC, MNHN

UCCC, Kuschel (1957) 
TABLA 2 (continuación)

\begin{tabular}{lll}
\hline ID Taxa & $\begin{array}{l}\text { Cuadrículas (en paréntesis, } \\
\text { extrapolación GAP) }\end{array}$ \\
\hline
\end{tabular}

279 Aegorhinus servillei Gay \& Sol.

280 Aegorhinus superciliosus (Guér.)

281 Aegorhinus suturalis (B1.)

285 Apocnemidophorus obsoletus (B1.)

286 Apocnemidophorus pruinosus (B1.)

288 Araucarietius viridans Kuschel

289 Araucarius minor (Kuschel)

291 Atrichonotus pacificus Kuschel

293 Callirhynchinus exquisitus F. \& G.

295 Chileudius varians (B1.)

298 Eisingius araucariae Kuschel

299 Eucalus tessellatus (B1.)

302 Geniocremnus villosus (B1.)

303 Germainiellus ovatus (Boh.)

305 Graphognathus leucoloma_(Boh.)

306 Hybreoleptops aureosignatus (B1.)

308 Hybreoleptops vestitus (B1.)

310 Hylaster ater (Pykull)

311 Hylurgus ligniperda (Fabr.)

312 Listroderes annulipes $\mathrm{B} 1$.

315 Listronotus bonariensis (Kuschel)

323 Neopachytychius squamosus Hustache

324 Neopsilorhinus variegatus (B1.)

327 Nototactus latirostris Kuschel

328 Omoides flavipes (B1.)

331 Parergus fascioliger (B1.)

332 Pentarthrum castaneum (B1.)

333 Platyaspistes marginalis Frahraeus

335 Polydrussus nothofagi Kuschel

336 Polydrussus roseus $\mathrm{Bl}$.

337 Rhopalomerus tenuirostris B1.

338 Rhyephenes goureaui (Gay \& Sol.)

339 Rhyephenes humeralis (Guér.)

340 Rhyephenes laterali (Guér.)

341 Rhyephenes maillei (Gay \& Sol.)

346 Strangaliodes albosquamosus Boh.

347 Strangaliodes mutuarius Kuschel

348 Tartarisus perforatipennis (B1.)

350 Tartarisus subfasciatus (B1.)

\section{Elateridae}

367 Deromecus griscens Cand.

369 Deromecus vulgaris Sol.

371 Elater decorus $\mathrm{G}$.

372 Lacon chilensis Sol.

373 Lynnyella gerhardtae Arias

374 Medonia punctatosulcata (Sol.)

375 Nyctophyxis ocellatus (G.)

376 Ovipalpus pubescens Sol.

377 Phanophorus perspicax (Guér.)

378 Podonema impressus Sol.

379 Pomachilius deromecoides (Schw.)

380 Semiotus luteipennis Guér.

381 Tibionema abdominalis (Guér.)
$36,45,60,68(35)$

UMCE, MNHN, Kuschel (1957)

$2,8,14,15,24,26,30,36$, $39,45,55,57,67,68,76,82$ $(25,35,56,75)$

$2,14,29,55$

2,14

19,24

68,72

24,68

$20,24,60,82$

30,31

$24,45,56(35,45)$

56,68

24,25 (14)

$2,14,24$

$14,16,24,26,29,33,39(15,25) \quad$ UCCC, Morrone (1993)

$2,14,15,16,17,18,19,24,26$,

$37,55,67(25)$

$2,16,24,36,45,68$

56,76

$15,20,24,36(25,14)$

$14,15,18,24,29,49$

29,68

$18,57,82$

18,35

$2,10,14,24,38$

$18,19,68$

2,26

17,31

2,18

$17,24,49$

$14,19,21,24,26,30,68$ (25)

30,74

45,68

39,68

$6,8,14,15,24,26,29,30,31$, $35,49,53(25)$

2,14

$14,15,24,45,53,67,68,82$

2,14

$18,24,26,31,35,37,48$ (36)

$30,31,52$

8,30

$24,36,45(35,45)$

$14,24,68$

$2,24,26,35$ (25)

31,68

18,31

24,67

$8,28,30,36,49$ (29)

18,24

7, 14, 19, 21, 24, 26, 30, 31,

$36,38,49(25,20)$

31,45

$24,45,47$

53,76

$14,17,24,26,35,41$ (25)
UCCC, Kuschel (1957)

UCCC, Kuschel (1957)

$\mathrm{UCCC}$

UCCC

UCCC, MNHN

UCCC, MNHN

MNHN

MNHN, Elgueta (1986)

UCCC, MNHN

Kuschel (2000)

UCCC

$\mathrm{UCCC}$

$\mathrm{UCCC}$

UCCC, UMCE, MNHN

MNHN, UCCC

UCCC

UCCC, Angulo (1996)

UCCC

MNHN

MNHN

UCCC

MNHN

UMCE

MNHN

UCCC, MNHN

$\mathrm{UCCC}, \mathrm{MNHN}$

UCCC, MNHN

MNHN

UMCE

UCCC

UCCC

UCCC

UCCC, Mendoza \& Jerez (2001),

Camus \& Barahona (2002)

$\mathrm{UCCC}$

UCCC, MNHN

MNHN

MNHN

UMCE, UCCC

UCCC

UCCC

MNHN, UCCC

Arias (2001)

UMCE

UCCC

UCCC

MNHN, UCCC

UCCC, UMCE

MNHN, UMCE

UCCC

UCCC, Angulo (1969-1970) 
TABLA 2 (continuación)

\begin{tabular}{llll}
\hline ID & Taxa & $\begin{array}{l}\text { Cuadrículas (en paréntesis, } \\
\text { extrapolación GAP) }\end{array}$ & Fuente \\
\hline $\begin{array}{l}\text { 9. Leiodidae } \\
\text { Dasypelates gracilis (Portev.) }\end{array}$ & 28,35 & MNHN, Jeannel (1962b), \\
425 & Eunemades chilensis Portev. & $20,25,35,41$ & $\begin{array}{l}\text { Newton \& Thayer (2003) } \\
\text { UCCC, Salgado (1991), } \\
\text { Colección, Newton \& Thayer (2003) }\end{array}$ \\
427 & Eupelates transversestrigosus (F \& G) & 24,28 & $\begin{array}{l}\text { UCCC, Jeannel (1962b), Salgado (1991), } \\
\text { Newton \& Thayer (2003) } \\
430\end{array}$ \\
Neopelatops chillanensis Jeannel & 31,72 & Jeannel (1962b), Newton \& Thayer (2003)
\end{tabular}

\section{Lucanidae}

439 Apterodorcus bacchus Hope \& Westwood 8, 14, 24, 26, 30, 35, 55,

440 Chiasognathus granti Stephens $\quad 18,76$

441 Chiasognathus mniszschi Thomson 30, 31

442 Pycnosiphorus arrugutti Weinreich 30, 31

443 Pycnosiphorus brevicollis Benesh 2,30

444 Pycnosiphorus caelatus (B1.)

445 Pycnosiphorus fasciatus (G.)

446 Pycnosiphorus lessoni (Buguet)

447 Pycnosiphorus magnificus Benesh

448 Pycnosiphorus rubrovittatus (B1.)

450 Sclerostomus cucullatus (B1.)

451 Streptocerus speciosus $\mathrm{F}$.

\section{Mordellidae}

460 Mordella castaneipennis $\mathrm{F}$ \& G

461 Mordella erythrura F \& G

462 Mordella fumosa F \& G

464 Mordella luctuosa Sol.

465 Mordella nana F \& G

466 Mordella ruboaxillaris $\mathrm{F} \& \mathrm{G}$

468 Mordella sericata F \& G

469 Mordella suturalis $\mathrm{F} \& \mathrm{G}$

470 Mordella vidua $\mathrm{F}$ \& $\mathrm{G}$

\section{Scarabaeidae}

481 Aphodius granarius (L.)

487 Athilia plebeya Burm.

488 Athlia rustica Er.

489 Aulacopalpus castaneus (Lap.)

490 Aulacopalpus ciliatus (Sol.)

492 Aulacopalpus viridis Sol.

493 Brachysternus prassinus Guér.

494 Brachysternus sinuatifrons $\mathrm{G}$.

496 Hylamorpha elegans (Burm.)

497 Macrodactylus crassipes $\mathrm{Ph}$.

498 Megathopa villosa Esch.

499 Oogenius kuscheli Gutierrez

500 Oryctomorphus bimaculatus Guér.

501 Oryctomorphus laevipennis $\mathrm{G}$.

503 Phytholaema herrmanni G.

504 Phytholaema mutabilis Sol.

505 Polymoneus brevicollis Esch.

509 Sericoides variegatus (G.)

510 Sericoides viridis (Sol.)

511 Tomarus villosus (Burm.)
$2,67,76$

$14,24,35,55,56,67,76(75,66)$

$20,30,31,35$

$2,24,26,29,35(25)$

$2,14,16,24,26,35,45,55$

$(15,25,56)$

$21,26,30,31$

$18,26,31,36$

$2,18,29,30(28,19)$

$17,26,45,68$

19,29

$2,8,18,19,25,26,30,31$,

$36,56,57$

$19,30,31$

$17,19,67$

$2,8,15,30,31,68$

$19,30,31$

$2,8,19,24,26,30,31,53$ (25)

$14,18,20,24,39,48$

$25,30,39,49,52,57$

$24,39,41$

$14,24,25,39,57$ (40)

2,24

$2,17,24,57$

$14,15,16,18,24,26,30,31$,

$35,39,41,57,60(17,25)$

$14,18,20,24,39,68$

$8,24,39$

30,31

$8,24,39$

11,74

$16,19,24,26,31,60,72,76(25)$

49,72

$14,18,24,57$

$17,18,24,39,60$

18,24

17,47

$17,24,31,36,45,53$ (35)

$14,17,18,19,24,25,30,35$, $39,48,49,57,60$
UCCC, UMCE

$\mathrm{UCCC}$

UCCC, MNHN

UCCC, MNHN

UCCC, MNHN

$\mathrm{UCCC}$

UCCC, MNHN

UCCC, Numhauser (1985)

UCCC

UCCC

UCCC, MNHN

UCCC, MNHN

UCCC

UMCE, MNHN

UCCC

UCCC, MNHN, UMCE

UCCC

UCCC, MNHN, UMCE

$\mathrm{UCCC}$

UCCC, MNHN

UCCC, UMCE

UCCC

UCCC

UCCC

UCCC

$\mathrm{UCCC}$

UCCC

UCCC, MNHN

UCCC

UCCC

MNHN

UCCC, Ovalle \& Solervicens (1980)

MNHN

$\mathrm{UCCC}$

$\mathrm{UCCC}$

UCCC

UCCC

UCCC

UMCE

UCCC, UMCE, MNHN

UCCC, MNHN

\section{Staphylinidae}

537 Achillia cosmoptera (B1.) 
TABLA 2 (continuación)

\begin{tabular}{|c|c|c|c|}
\hline ID & Taxa & $\begin{array}{l}\text { Cuadrículas (en paréntesis, } \\
\text { extrapolación GAP) }\end{array}$ & Fuente \\
\hline 540 & Achillia frontales Jeannel & 31,72 & Jeannel (1962a) \\
\hline 549 & Atheta obscura (Sol.) & 11,18 & Pace $(2000)$ \\
\hline 550 & Atheta obscuripennis (Sol.) & 18,31 & Pace (2000) \\
\hline 562 & Bolitobius seriaticollis & 20,36 & $\mathrm{UCCC}$ \\
\hline 565 & Cheilocolpus pyrostoma (Sol.) & $20,35,36$ & UCCC \\
\hline 567 & Creophilus erythrocephalus (Fabr.) & $24,31,55,57$ & UCCC \\
\hline 568 & Creophilus maxillosus (L.) & $17,24,76$ & $\mathrm{UCCC}$ \\
\hline 577 & Golasa microcephala (Reitter) & 31,72 & Jeannel (1962a) \\
\hline 581 & Lamprostiba schwabei (Bernhauer) & $11,18,31$ & Pace $(2000)$ \\
\hline 582 & Leptoglossula biimpressa (Sol.) & $11,18,31$ & Pace $(2000)$ \\
\hline 583 & Leptoglossula mixta Pace & $11,18,31$ & Pace $(2000)$ \\
\hline 584 & Leptoglossula robusta Pace & $11,18,31$ & Pace (2000) \\
\hline 585 & Leptoglossula sculpticollis (Fauvel) & $11,18,31$ & Pace (2000) \\
\hline 593 & Ophioglossa araucana Fauvel & $11,18,31,76$ & Pace $(2000)$ \\
\hline 594 & Ophioglossa franzi Pace & 11,31 & Pace (2000) \\
\hline 595 & Pagla anthracina (F. \& G.) & $11,18,31$ & Pace (2000) \\
\hline 597 & Pagla daccordii Pace & 18,31 & Pace $(2000)$ \\
\hline 603 & Paractium mochae Franz & 16,82 & Elgueta \& Cekalovic (1999) \\
\hline 611 & Placusa chilensis Fauvel & 57,76 & Pace $(2000)$ \\
\hline 612 & Plesiomalota fasciatipennis (F. \& G.) & $11,18,31$ & Pace (2000) \\
\hline 613 & Plesiomalota hispidula (Fauvel) & $11,18,31$ & Pace (2000) \\
\hline 614 & Plesiomalota mehuinensis Pace & $11,18,31$ & Pace $(2000)$ \\
\hline 615 & Plesiomalota merula (Fauvel) & $11,18,31$ & Pace (2000) \\
\hline 618 & Prosoponotha chilensis Pace & $11,18,31$ & Pace $(2000)$ \\
\hline \multirow[t]{2}{*}{631} & Tyropsis cavifrons Raffray & 31,72 & Jeannel (1962a) \\
\hline & 14. Tenebrionidae & & \\
\hline 635 & Allecula chilensis Campbell & 30,68 & UCCC \\
\hline 636 & Allecula pallidicollis (F.) & 56,68 & UCCC \\
\hline 640 & Blapstinus punctulatus Sol. & $7,8,14,24,29,30,38,41,82$ & UCCC \\
\hline 641 & Callyntra carbonaria (Phil. \& Phil.) & $30,31,53,72,73(64)$ & Flores \& Vidal (2000) \\
\hline 642 & Callyntra inflata (Peña) & $2,16,29,31,45$ & Flores \& Vidal (2000) \\
\hline 644 & Callyntra paulseni $\mathrm{F}$. & $2,16,53$ & Flores \& Vidal (2000) \\
\hline 646 & Callyntra rossi (Kulzer) & $20,24,31,56,68$ & UCCC, MNHN, Flores \& Vidal (2000) \\
\hline 647 & Callyntra rugosa (Waterhouse) & 11,31 & UMCE \\
\hline 648 & Callyntra servillei Sol. & 2,24 & Flores \& Vidal (2000) \\
\hline 690 & Epipedonota rugosa Waterhouse & 31,53 & UMCE \\
\hline 649 & Eucaliga sanguinicollis $\mathrm{F}$. & $10,18,31,68$ & UCCC \\
\hline 654 & Heliofugus arenosus Guér. & $7,15,20,24(14,25)$ & UCCC \\
\hline 656 & Heliofugus germaini Freude & $14,30,31,68$ & UCCC \\
\hline 657 & Heliofugus impressus Guér. & $\begin{array}{l}2,8,30,31,41,45,55,56, \\
57,68,72(67)\end{array}$ & UCCC, MNHN \\
\hline 659 & Heliofugus rossi Freude & $56,60,68$ & Freude (1987) \\
\hline 660 & Heliofugus tenuipunctatus Phil. & $10,30,31$ & UCCC \\
\hline 661 & Homocyrtus bonni $(\mathrm{G})$. & $17,24,26,36,60(25)$ & UCCC \\
\hline 662 & Homocyrtus dives $\mathrm{G}$. & $14,20,24,29,39,45,60(35)$ & UCCC \\
\hline 663 & Nyctelia nodosa $\mathrm{G}$ & $17,24,26,36,60(25)$ & $\mathrm{UCCC}$ \\
\hline 665 & Nycterinus abdominalis Esch. & $14,24,82$ & UCCC, Peña (1971) \\
\hline 666 & Nycterinus angusticollis Phil. & $6,14,17,24,29,30,53,55$ & UCCC, Peña (1971) \\
\hline 668 & Nycterinus laevigatus Phil. & $24,29,33,39,45,53,82$ & UCCC, Peña (1971) \\
\hline 671 & Nycterinus thoraxicus Esch. & $2,7,15,26,29,35,57(16)$ & UCCC, MNHN, Peña (1971) \\
\hline 672 & Nyctopetus argentinus Freude & 24,53 & $\mathrm{UCCC}$ \\
\hline 674 & Nyctopetus tenebrioides Guér. & $\begin{array}{l}15,24,39,45,52,53,56 \\
68,72(14,25)\end{array}$ & UCCC \\
\hline 675 & Oligocara nitidum Sol. & $\begin{array}{l}2,7,8,11,14,15,20,24, \\
26,30,31,35,41,55,56, \\
57,68,72,82(25)\end{array}$ & UCCC, MNHN, UMCE \\
\hline 676 & Phaleria gayi Lap. & 15,35 & UCCC \\
\hline 677 & Phalerisida maculata Kulzer & $2,15,24,35(14)$ & UCCC, Camus \& Barahona (2002) \\
\hline 678 & Praocis costata Sol. & $15,24,39,35,45,55$ & UCCC \\
\hline 679 & Scotobius bullatus Curtis & $\begin{array}{l}8,14,17,18,20,24,29 \\
33,55(19)\end{array}$ & UCCC \\
\hline
\end{tabular}




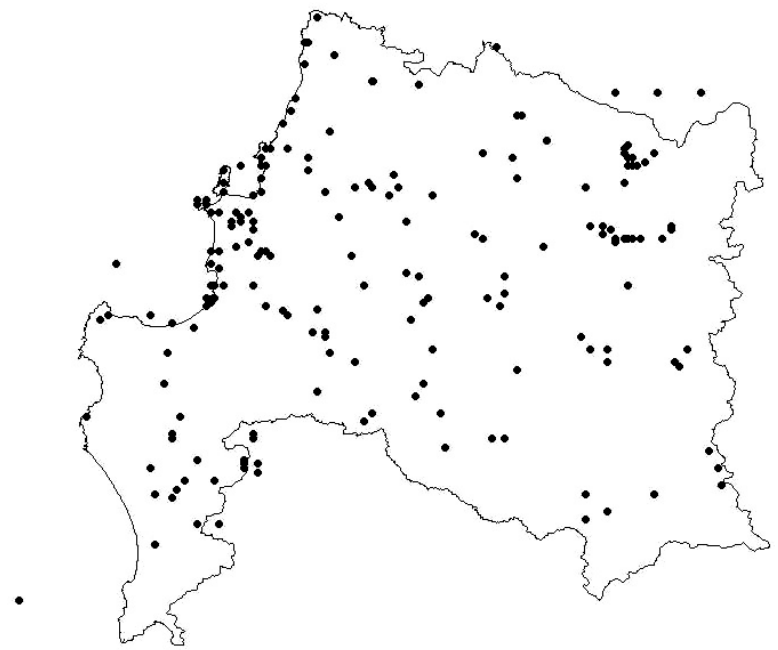

Fig. 2: Distribución y puntos de registros de las especies de coleópteros en la Región del Biobío. Distribution and point records of beetles species in the Biobío Region.

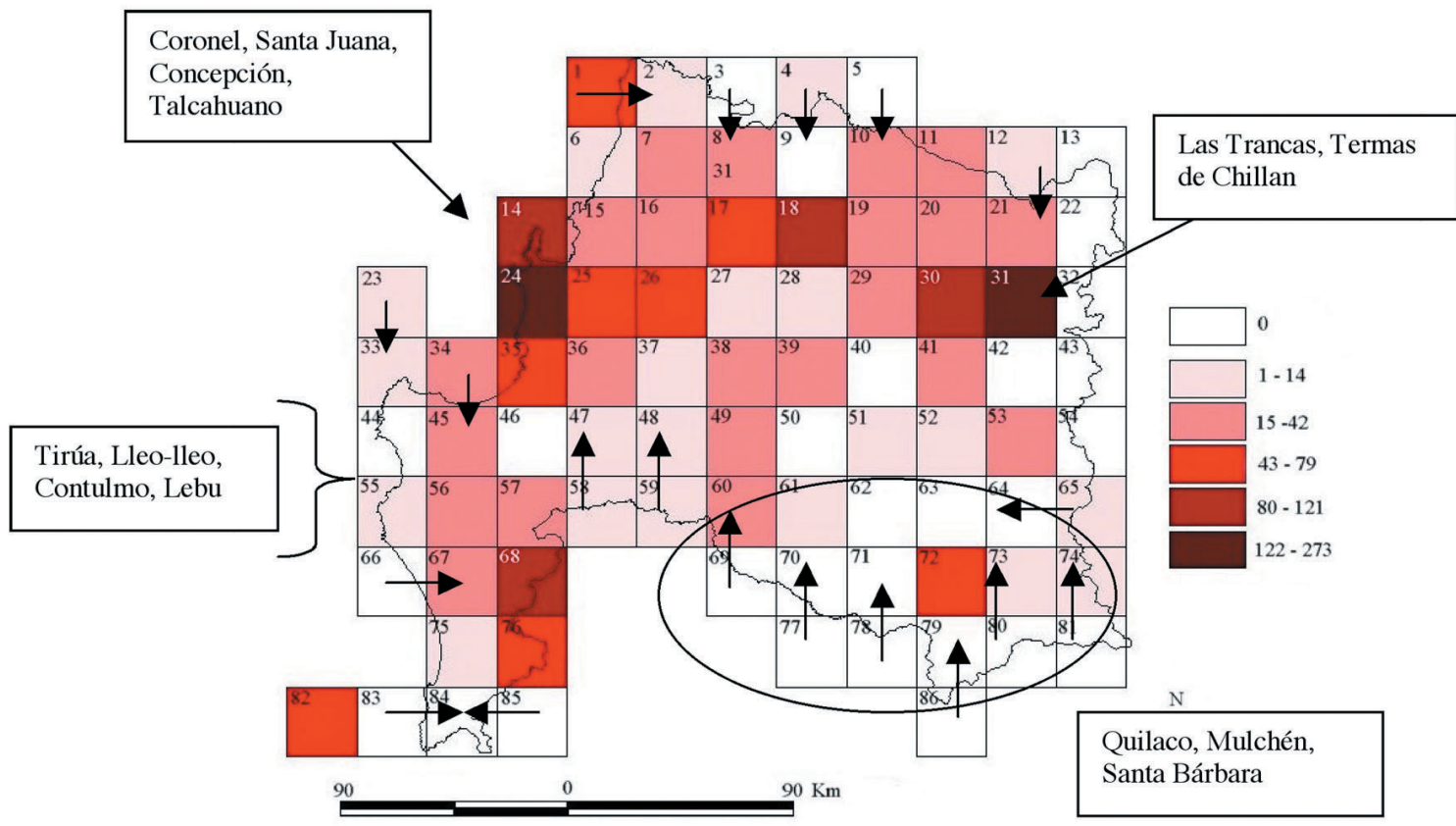

Fig. 3: Mapa digitalizado de la Región del Biobío dividido en cuadrículas. Se muestra la riqueza de especies por cuadrícula.

Digitalized map of the Biobío Region divided in quadrants. The species richness for quadrants is showed.

Determinación de áreas de concentración de especies

En el PAE (Fig. 5), la búsqueda heurística generó 1.000 árboles igualmente parsimoniosos $(\mathrm{L}=1.018 ; \mathrm{IC}=0,28 ; \mathrm{IR}=0,34)$ a partir de los cuales se obtuvo un árbol de consenso estricto
$(\mathrm{L}=1.076 ; \mathrm{IC}=0,27 ; \mathrm{IR}=0,29)$. Por otra parte, el análisis bootstrap generó 2002 árboles $(\mathrm{L}=$ 1.067; IC $=27$ y IR = 30). El árbol de consenso estricto muestra grupos no resueltos y una politomía, por lo cual no se observó un patrón marcado de agrupación de cuadrículas. Sin embargo, se obtuvieron 3 áreas de agrupación de 
especies: Área 1: incluye la agrupación de las cuadrículas $25+(14+24)$ con Acalles moestus (ID: 270) y Eucalus tessellatus (ID: 299) con un $75 \%$ de apoyo bootstrap. Área 2: incluye el conjunto de cuadrículas $8+(30+31)$ con $71 \%$ de apoyo bootstrap sustentada por la presencia de dos especies sinapomórficas: Conognatha souverbii (ID: 46) y Calendyma chilensis (ID: 215). El conjunto $30+31$ fue sustentado con un $93 \%$ de apoyo bootstrap con ocho especies sinapomórficas: Conognatha azurea (ID: 35), Dactylozodes semivittata (ID: 52), Planopus laniniensis (ID: 154), Sybilla livida (ID: 161), Callirhynchinus exquisitus (ID: 293), Chiasognathus mniszschi (ID: 441), Pycnosiphorus arrugutti (ID: 442) y Macrodactylus nigrinus (ID: 497). Área 3: incluye el conjunto de cuadrículas $56+68$ con un $94 \%$ de apoyo bootstrap con dos especies sinapomórficas: Eisingius araucariae (ID: 298) y Allecula pallidicollis (ID: 636).

Al desactivar las especies sinapomórficas, el análisis PAE-PCE agrupó las cuadrículas: 11+ $(18+31)$ con 11 especies sinapomórficas, todas pertenecientes a Staphylinidae: Lamprostiba schwabei (581), Leptoglossula biimpresa (582), Leptoglossula mixta (583), Leptoglossula robusta (584), Leptoglossula sculpticollis (585), Pagla anthracina (595), Plesiomalota fasciatipennis (612), Plesiomalota hispidula (613), Plesiomalota mehuinensis (614), Plesiomalota merula (615) y Prosoponotha chilensis (618) con un $57 \%$ de apoyo bootstrap y las cuadrículas $18+31$ con tres especies que sustentan la unión Lynnyella gerhardtae (373), Atheta obscuripennis (550) y Pagla daccordii (597) con $57 \%$ de apoyo bootstrap. Además, se agruparon las cuadrículas $36+(45+(35+(14$
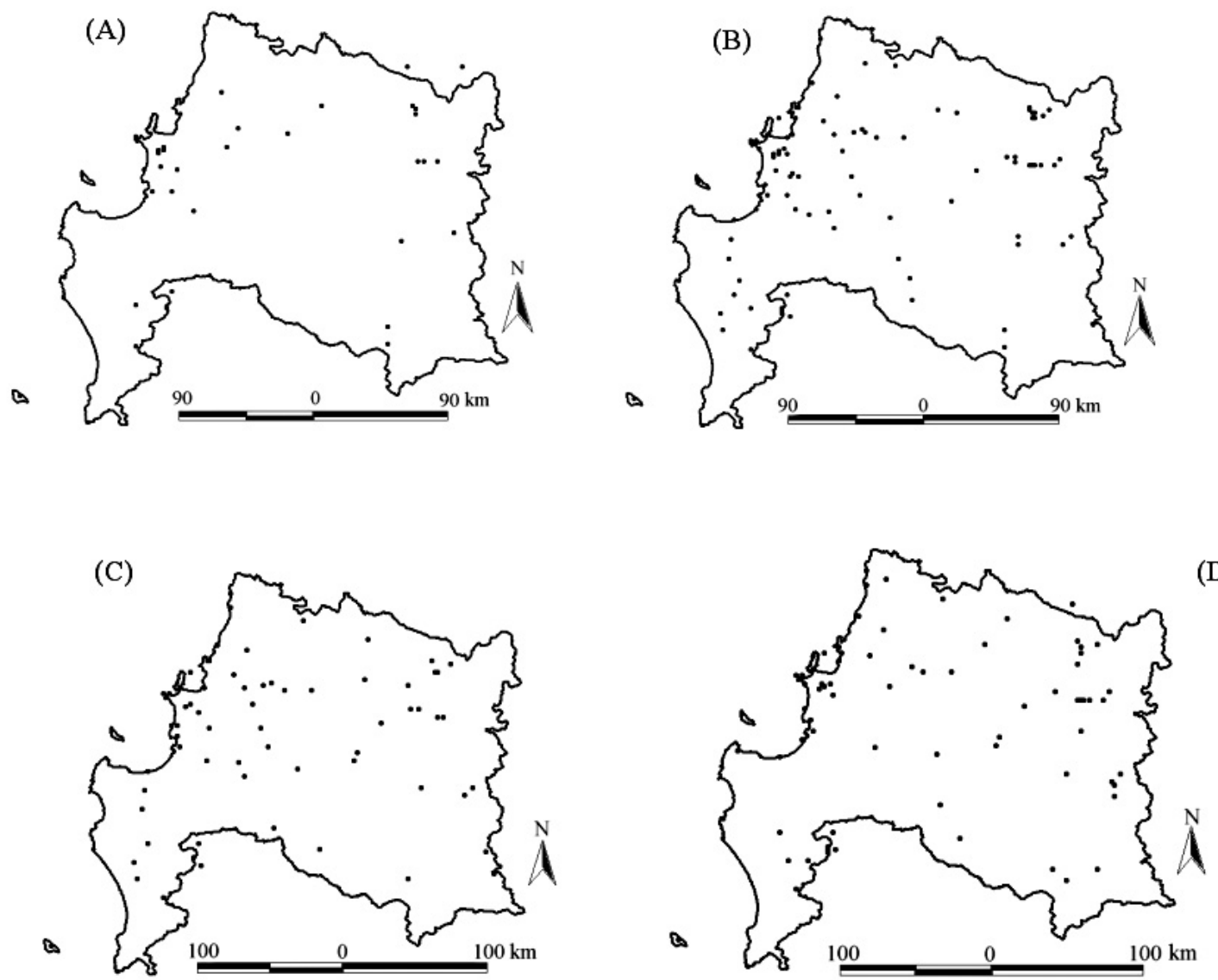

(D)

Fig. 4: Localidades de distribución para familias y especies de coleópteros en la Región del Biobío. (A) Staphylinidae (B) Curculionidae (C) Chrysomelidae (D) Tenebrionidae.

Distribution localities for families and species of beetles in the Biobío Region. (A) Staphylinidae (B) Curculionidae (C) Chrysomelidae (D) Tenebrionidae. 
+24))) mediante las especies Stenomela pallida (ID: 213) y Deromecus griscens (ID: 367) con un $55 \%$ de apoyo "bootstrap" (2.002 árboles con $\mathrm{L}=1.049 ; \mathrm{IC}=26$; IR: 29). Luego de las siguientes repeticiones no se observaron más grupos resueltos.

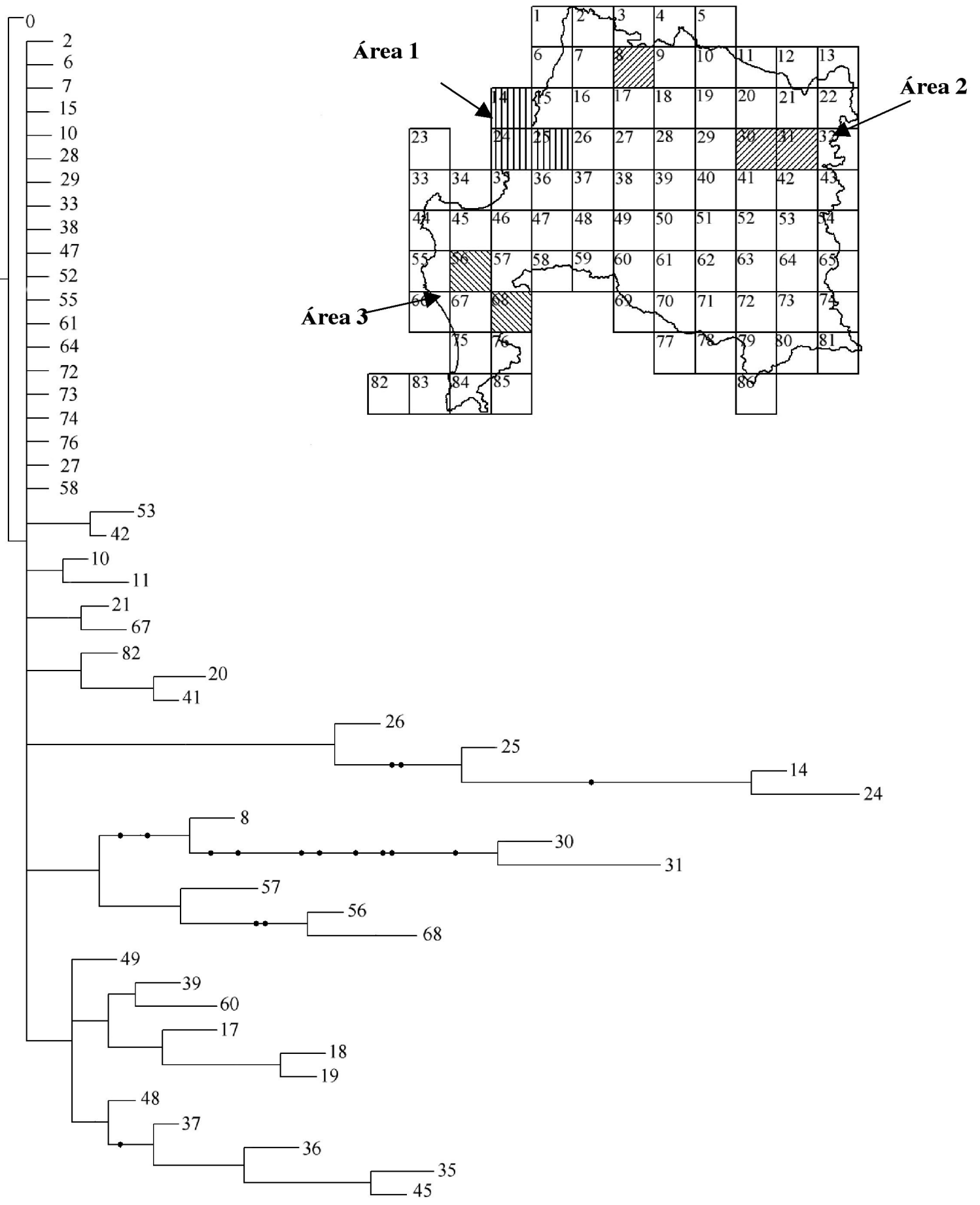

Fig. 5: Árbol de consenso estricto y áreas de concentración de especies de coleópteros obtenido mediante PAE para la Región del Biobío.

Strict consensus tree and concentration areas of beetles obtained by PAE for the Biobío region. 
Relación entre áreas de concentración de especies, formaciones vegetales y áreas silvestres protegidas

En la Fig. 6A, 6B y 6C se observa que las áreas de concentración de especies obtenidas en el PAE están relacionadas en cierta medida con las formaciones vegetales descritas para la región por Gajardo (1994) (Comisión Nacional del Medio Ambiente, Biobío 2003). El área 1 forma parte, principalmente, de las formaciones esclerófilas costeras de Concepción (Fig. 6A). El área 2 está ubicada entre el límite sur del bosque maulino y las formaciones vegetales de carácter caducifolio y de estepa que se extienden hasta la Región de la Araucanía (Fig. 6B). Finalmente el área 3 forma parte de la Cordillera de Nahuelbuta (Fig. 6C), sector en el que predomina la vegetación esclerófila en la vertiente oriental y bosque caducifolio en las partes más altas. Ninguna de las áreas obtenidas con el PAE, están representadas en alguna de las áreas silvestres protegidas establecidas para la región del Bíobío por el Sistema Nacional de Áreas Silvestres Protegidas del Estado (SNASPE) (Fig. 6D).

En el análisis PAE-PCE, una de las áreas agrupadas corresponde al sector precordillerano andino de Nuble y la otra al sector costero, en el cual se encuentran especies de coleópteros relacionadas con vegetación del bosque esclerófilo y parches de bosque caducifolio de Concepción (Fig. 6A y 6B).
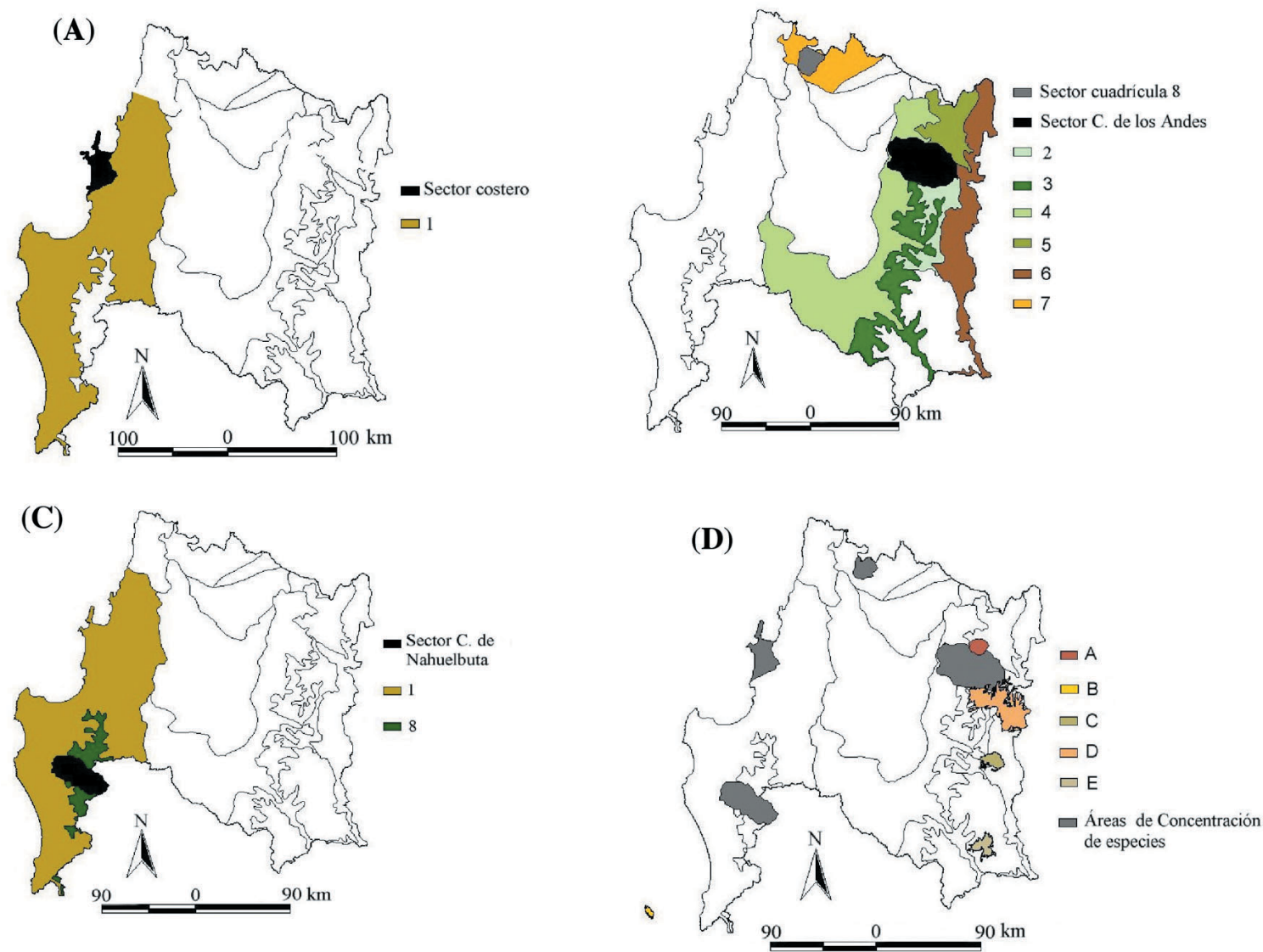

Fig. 6: Distribución de especies, relación con las formaciones vegetales y Áreas Silvestres Protegidas por el Estado. (A) sector costero, (B) sector cordillera de los Andes, (C) sector cordillera de Nahuelbuta, (D) distribución de especies según Áreas Silvestres Protegidas.

Distribution of species in relation with plant formations and the Protected Wild Areas. (A) Coastal sector, (B) Andean mountain range sector, (C) coastal mountain range of Nahuelbuta and, D) distribution of species according to Protected Wild Areas.

1: Bosque caducifolio y esclerófilo de Concepción; 2: bosque caducifolio alto-andino de Chillán; 3: bosque caducifolio andino del Biobío; 4: bosque caducifolio de la frontera; 5: bosque caducifolio de la montaña; 6: estepa alto-andina boscosa; 7: matorral espinoso del secano interior; 8: bosque alto-montano de Nahuelbuta; A: Reserva Nacional Huemules de Niblinto; B: Reserva Nacional Isla Mocha; C: Parque Nacional Laguna del Laja; D: Reserva Nacional Nuble; E: Reserva Nacional Ralco. 


\section{DISCUSIÓN}

Chile se caracteriza por su fauna y flora altamente endémicas (Simonetti 2000), un legado de los cambios climáticos y vegetacionales ocurridos durante el Terciario y migraciones de la vegetación durante el último ciclo glacial (Villagrán \& Armesto 2005). Evidencias de estas migraciones se encuentran en la Región del Biobío, que desde el punto de vista biogeográfico constituye una zona de contacto entre biotas provenientes de las ecorregiones Matorral de Chile y Bosque Templado de Valdivia (Teneb et al. 2004). Estas ecorregiones se caracterizan por sus altos niveles de endemismo y riqueza florística (Villagrán \& Armesto 2005) y faunística. Nuestros resultados, sin embargo, muestran para coleópteros, una baja riqueza específica, ya que las 664 especies registradas, representan solo el $17 \%$ del total de especies descritas para el país. Por el contrario para familias $(55 \%)$ y géneros $(30 \%)$ la diversidad es mayor, ya que ambas categorías taxonómicas representan alrededor de la cuarta parte del total descrito para el país.

La menor diversidad específica de coleópteros encontrada podría ser el resultado de factores histórico-geográficos y antropogénicos que han afectado a la región. En aspectos biogeográficos, la fauna chilena de coleópteros de Chile Central y Región Subantártica es mayoritariamente endémica y se caracteriza por la presencia de numerosas familias y géneros monotípicos (Solervicens 1995) como es el caso de Cupedidae con Prolixocupes latreille, Tetratomidae con Serropalpus valdivianus (Elgueta 2000a) y Chrysomelidae con Stenomela pallida y Jansonius aeneus (Jerez 2005), entre otros. Por otro lado, la alteración antropogénica representada por la degradación de ambientes naturales, producto de la introducción de especies exóticas (Cavieres et al. 2005), uso de suelo para ganadería y agricultura, y el emplazamiento de zonas urbanas tal como lo señalan Polymeris (1995), Cisternas et al. (1999) y Arroyo (2002), también es un factor que puede explicar la baja diversidad de especies y la ausencia de registros en muchas cuadrículas.

En relación con la presencia de endemismos, existen géneros y/o especies endémicas a una provincia biogeográfica y no a esta región en particular. Este es el caso de Callyntra rossi Kulz. tenebriónido endémico para la cordillera de Nahuelbuta (Flores \& Vidal 2000) y de Cnemalobus germaini Kulz. carábido cuya distribución está restringida a la provincia Subantártica (Roig-Juñent 1994).

Sin embargo, muchos taxa presentan rangos de distribución discontinuos y restringidos a las formaciones vegetales en que habitan, por lo que podrían considerarse como taxa subendémicos (Rovito et al. 2004). Por ejemplo, el crisomélido Stenomela pallida presenta un patrón de distribución discontinuo en sectores húmedos de la cordillera de la costa y está asociada exclusivamente a Myrtáceas (Jerez 1996), sin embargo, en la región del Bíobío está presente solamente en formaciones esclerófilas e higrófilas costeras, lo que explica su presencia en pocas cuadrículas. Otros casos son el de Eurymetopum frigidum y $E$. semirufum, cléridos asociados a relictos de bosques de Nothofagus al inicio de la cordillera de Nahuelbuta y en la cordillera de Chillán, que han extendido su rango distribucional a bosques de pantano, esclerófilos o matorrales xerófilos más al norte (Solervicens 2005).

Dentro de las especies más representativas para la región, destacan Apterodorcus bacchus, Aegorhinus superciliosus, Astylus trifasciatus, Ceroglossus chilensis, Oligocara nitidum y Procalus mutans, taxa de formas vistosas y de gran tamaño, características que facilitan su visualización y captura. En relación con los patrones de distribución, Curculionidae, Tenebrionidae y Chrysomelidae se distribuyen en toda la región, familias que según Peña (1996) poseen un amplio rango de hábitos alimenticios y de ambientes donde habitan. Por el contrario, Staphylinidae presenta una distribución restringida solamente a algunos sectores como sector costero de Concepción, precordillera de Chillán y Cordillera de Nahuelbuta, la que podría ser explicada por la destrucción de sus hábitats en la depresión intermedia y zonas intermedias.

El análisis PAE muestra la agrupación de tres áreas importantes de concentración de especies de coleópteros. El área 1 corresponde a los últimos remanentes del bosque costero esclerófilo y caducifolio de Concepción, sectores que a pesar de estar altamente intervenidos (Ibarra-Vidal et al. 2005), 
albergan el mayor número de especies de coleópteros descritas para la región; esto justificaría su conservación per se. El área 2 forma parte de los bosques caducifolios alto andino de Chillán, altoandino del Biobío, de la frontera y estepa altoandina boscosa. Esta heterogeneidad vegetacional, explicaría la gran diversidad de coleópteros allí encontrados. El área 3 corresponde a una parte de la Cordillera de Nahuelbuta, unidad biogeográfica (O’Brien 1971, Artigas 1975) caracterizada por diversos taxa endémicos y que representa el límite septentrional de la distribución de algunas especies de coleópteros asociadas principalmente con Araucaria como es el caso de Eisingius araucariae (Kuschel 2000) y de especies asociadas a la región subantártica (Jerez 2005).

En cuanto a estrategias de conservación, las áreas de concentración de especies encontradas no están incluidas dentro de las áreas silvestres protegidas por el Estado (Comisión Nacional del Medio Ambiente Biobío 2003). Esto se debería por una parte a la escasa proporción de terreno protegida, a su alto grado de deterioro histórico y a la fragmentación, haciendo difícil la conservación y/o restauración de los ambientes (Ibarra-Vidal et al. 2005). Por otra parte, en Chile, solo los animales más vistosos y menos abundantes, han sido utilizados en los estudios de conservación (Vergara \& Jerez 2005) ${ }^{1}$. Si bien los insectos son catalogados como indicadores de ambientes, no existe una clara definición en términos políticos y/o científicos de su estado de conservación en el país. Esperamos que en un futuro próximo se desarrollen instancias que sitúen a los coleópteros dentro de estas estrategias, proponiendo especies que por su singularidad contribuyan a determinar la protección de algunos ambientes.

\section{AGRADECIMIENTOS}

A Mario Elgueta del Museo Nacional de Historia Natural (MNHN), Chile y a Jaime Solervicens del Instituto de Entomología, Universidad Metropolitana de Ciencias de la

${ }^{1}$ VERGARA O \& V JEREZ (2005) La diversidad de coleópteros en la Región del Biobío y su aplicación en propuestas de gestión ambiental. Libro de Resúmenes XXVII Congreso Nacional de Entomología, Valdivia, Chile: 15 . educación (UMCE), Chile, por su disponibilidad para la revisión de colecciones de referencia; a Lohengrin Cavieres del Departamento de Botánica, Universidad de Concepción, Bolívar Ruiz, Director Comisión Nacional del Medio Ambiente (CONAMA) Biobío y Mauricio Aguayo, Centro EULAChile por el material digital; a Jaime Pizarro, Laboratorio de Entomología, Universidad de La Serena, Tomás Moore, Juan José Morrone del Museo de Zoología, Universidad Nacional Autónoma de México (UNAM), Sergio RoigJuñent, Instituto Argentino de Investigaciones en Zonas Áridas (IADIZA, CRICYT), Alfred Newton, Associate Curator Zoology Department/Insect Division Field Museum of Natural History, USA, y a José Mondaca, Servicio Agrícola y Ganadero, a todos ellos por las sugerencias y actualizaciones taxonómicas. Finalmente a Audrey Grez, editora asociada de la Revista Chilena de Historia Natural y a los revisores anónimos por sus valiosas sugerencias. Este trabajo fue financiado por el proyecto DIUC $\mathrm{N}^{\mathrm{o}}$ 203.113.061-1.0 de la Dirección de Investigación, Universidad de Concepción.

\section{LITERATURA CITADA}

ANGULO O (1969-1970) Descripción de la larva y pupa de Tibionema abdominalis (Guérin) (Coleoptera: Elateridae). Boletín de la Sociedad de Biología de Concepción (Chile) 42: 307-311.

ANGULO O (1996) Caracteres externos para separar los sexos del escolito rubio del pino, Hylurgus ligniperda (Fabricius) (Coleoptera: Scolytidae). Gayana Zoología (Chile) 60: 69-72.

ARIAS E (2000) Coleópteros de Chile. Litografía Valente, Santiago, Chile. 209 pp.

ARIAS E (2001) Lynnyella, a new genus of click beetles from central and southern Chile (Coleoptera: Elateridae). Gayana Zoología (Chile) 65: 137-148.

ARROYO MTK (1999) Criterios e indicadores para la conservación de la biota en ecosistemas mediterráneos. Revista Chilena de Historia Natural 72: $473-474$

ARROYO MTK (2002) Diversidad biológica. http:// www.centrogeo.org.mx/unep/documentos/Chile/ CHILEbiodiver, http://www.derecho.uchile.cl/cda/ d ocumentos / archivos / Biodiversidadt/ BioseguridadChile

ARTIGAS JN (1969-1970) Descripción de larva y pupa de Chenoderus testaceus (Blanchard) (ColeopteraCerambycidae). Boletín de la Sociedad de Biología de Concepción (Chile) 42: 245-250.

ARTIGAS JN (1975) Introducción al estudio por computador de las áreas zoogeográficas de Chile continental basado en la distribución de 903 especies de animales terrestres. Gayana Miscelánea (Chile) 4: 3-25. 
ASKEVOLD I \& L LESAGE (1990) A taxonomic revision of the genus Jansonius Baly 1878: taxonomic confusion and tribal reclassification (Coleoptera: Chrysomelidae; Eumolpinae). Revista Chilena de Entomología 18: 29-37.

BELLAMY CH \& T MOORE (1990) A review of the tribe Mendizabaliini Cobos with the addition of new taxa (Coleoptera: Buprestidae). Revista Chilena de Entomología 18: 39-48.

BRIONES R \& V JEREZ (2004) Coleópteros asociados al follaje y fenología de Lithrea caustica (Mol) (Anacardiaceae) en un fragmento de bosque costero, VIII Región, Chile. Gayana Zoología (Chile) 68: 43-52.

CAMUS P \& R BARAHONA (2002) Insectos del Intermareal de Concepción, Chile: perspectivas para la investigación ecológica. Revista Chilena de Historia Natural 73: 793-803.

CAVIERES L, M MIHOC, A MARTICORENA, C MARTICORENA, O MATTHEI \& F SQUEO (2001) Determinación de áreas prioritarias de conservación: análisis de parsimonia de endemismos (PAE) en la flora de la IV Región de Coquimbo. En: Squeo F, G Arancio \& J Gutiérrez (eds) Libro rojo de la flora nativa y de los sitios prioritarios para su conservación: Región de Coquimbo: 159-170. Ediciones Universidad de la Serena, La Serena, Chile.

CAVIERES L, M ARROYO, P POSADAS, C MARTICORENA, O MATTHEI, R RODRÍGUEZ, F SQUEO \& G ARANCIO (2002) Identification of priority areas for conservation in an arid zone: application of parsimony analysis of endemicity in the vascular flora of the Antofagasta region, northern Chile. Biodiversity and Conservation 11: 1303-1311

CAVIERES L, M MIHOC, A MARTICORENA, C MARTICORENA, C BAEZA \& MTK ARROYO (2005) Flora vascular de la Cordillera de la Costa en la Región del Biobío: riqueza de especies, géneros, familias y endemismos. En: SmithRamírez, JJ Armesto \& C Valdovinos (eds) Historia, biodiversidad y ecología de los bosques costeros de Chile: 245-552. Editorial Universitaria, Santiago, Chile.

CERDA M (1973) Nueva tribu de Cerambycinae (Coleptera: Cerambycidae). Revista Chilena de Entomología 7: 115-122.

CISTERNAS M, P MARTÍNEZ, C OYARZÚN \& $\mathrm{P}$ DEBELS (1999) Caracterización del proceso de reemplazo de vegetación nativa por plantaciones forestales en una cuenca lacustre de la Cordillera de Nahuelbuta, VIII Región, Chile. Revista Chilena de Historia Natural 72: 661-669.

CONAF-CONAMA-BIRP (eds) (1999) Catastro y evaluación de recursos vegetacionales nativos de Chile. Informe Regional Octava Región. Universidad Austral de Chile, Pontificia Universidad Católica de Chile y Universidad Católica de Temuco. 130 pp.

CONAMA-BIOBÍO (eds) (2003) Estrategia nacional de biodiversidad. Gobierno de Chile, Comisión Nacional de Medio Ambiente, Programa de las Naciones Unidas para el Desarrollo, Concepción, Chile. $21 \mathrm{pp}$

CORTÉS X, V JEREZ \& R GALLEGUILLOS (1993) Estudio electroforético de dos especies simpátricas y sintópicas del género Procalus Clark, 1865 (Coleoptera: Chrysomelidae). Revista Chilena de Entomología 20: 43-48.
DIEPENBROEK M, H GROBE \& R SIEGER (2000) PanMap; www.pangea/software/panma.

ELGUETA M (1986) Redescubrimiento de Callirhynchinus exquisitus (Faimaire \& Germain, 1861) (Coleoptera: Curculionoidea; Belidae). Revista Chilena de Entomología 14: 99-102.

ELGUETA M (1995) Coleoptera. En: Simonetti J, M Arroyo, A Spotorno \& E Lozana (eds) Diversidad biológica de Chile: 246-252. Comité Nacional de Diversidad Biológica, Comisión Nacional de Ciencia y Tecnología, Santiago, Chile.

ELGUETA M (2000a) Coleoptera de Chile. En: MartínPiera F, J Morrone \& A Melic (eds) Hacia un proyecto Ciencia y Tecnología para el Desarrollo (CYTED) para el inventario y estimación de la diversidad entomológica en Iberoamérica: 145-154. PRIBES-2000, Sociedad Entomológica Aragonesa (SEA), Zaragoza I.

ELGUETA M (2000b) Dos especies nuevas de Aegorhinus (Coleoptera: Curculionidae: Aterpini) de Chile. Acta Entomológica Chilena 24: 7-18.

ELGUETA M \& T CEKALOVIC (1999) Curculionidae (Insecta: Coleoptera) de la isla Mocha, Arauco, Chile. Museo Nacional de Historia Natural, Noticiario Mensual (Chile) 335: 7-10.

ESCALANTE T \& J MORRONE (2003) ¿Para que sirve el análisis de parsimonia de endemismos? En: Llorente J \& J Morrone (eds) Una perspectiva latinoamericana de la biogeografía: 167-172. Facultad de Ciencias. Universidad Nacional Autónoma de México, Distrito Federal, México.

ESRI (1996) Software ARCVIEW-GIS 3.2. Published by the Environmental Systems Research Institute, Iinc., Redlands, New York, USA. 340 pp.

FATTORINI S (2002) Biogeography of the tenebrionid beetles (Coleoptera, Tenebrionidae) on the Aegean Islands (Greece). Journal of Biogeography 29: 4967.

FICHET T \& J BARRIGA (1990) Observaciones biológicas sobre Holopterus annulicornis F. Philippi, 1859 (Coleoptera: Cerambycidae). Revista Chilena de Entomología 18: 61-65.

FLORES G \& P VIDAL (2000) Revalidation and systematic revision of the Chilean genus Callyntra Solier (Coleoptera: Tenebrionidae). Annals of the Entomological Society of America 93: 1052-1075.

FREUDE H (1987) Neve Heliofugus Guérin, 1830 (Coleoptera: Tenebrionidae) und Eine Bestimmungstabelle Der Bisher Bekannten arter der Gattung. Revista Chilena de Entomología 15: 7-12.

GAJARDO R (1994) La vegetación natural de Chile. Clasificación y distribución geográfica. Editorial Universitaria, Santiago, Chile. 165 pp.

GARCÍA-BARROS E (2003) Mariposas diurnas endémicas de la Región Paleártica occidental: patrones de distribución y su análisis mediante parsimonia (Lepidoptera, Papilionoidea). Graellsia 59: 233-258.

GOLOBOFF P (1993) NONA version 2.0 (Windows). Computer program distributed by the author.

GREZ A, P MORENO \& M ELGUETA (2002) Coleópteros (Insecta: Coleoptera) epígeos asociados al bosque maulino y plantaciones de pino aledañas. Revista Chilena de Entomología 29: 5-18.

INSTITUTO NACIONAL DE ESTADÍSTICA (2002) Censo de población y vivienda 2002. Instituto Nacional de Estadística, Santiago, Chile; http:// www.censo2002.cl. http://www.ine.cl

IBARRA-VIDAL H, C SEPÚLVEDA, D SAAVEDRA \& E MALDONADO (2005) Propuestas de 
conservación de los bosques nativos en la cordillera costera del Maule y Bíobío (VII y VIII regiones). En: Smith-Ramírez C, JJ Armesto \& CC Valdovinos (eds) Historia, biodiversidad y ecología de los bosques costeros de Chile: 617-631. Editorial Universitaria, Santiago, Chile.

JEANNEL R (1962a) Les Pselaphides de la Paleantarctide occidentale. En: Delamare CL \& E Rapoport (eds) Biologie de 1'Amérique australe: 295-480. Volumen I. Editions du Centre National de la Recherche Scientifique du France et Consejo National de Investigaciones Científicas y Técnicas, Paris, Francia.

JEANNEL R (1962b) Les Silphidae, Leiodidae, Camiaridae et Catopidae de Paleantarctide occidentale. En: Delamare CL \& E Rapoport (eds) Biologie de 1'Amérique australe: 482-655. Volumen I. Editions du Centre National de la Recherche Scientifique du France et Consejo National de Investigaciones Científicas y Técnicas, Paris, Francia.

JEREZ V (1988) Estados inmaduros y biología de Lysathia atrocyanea (Phil \& Phil. 1864) (Chrysomelidae, Alticinae). Boletín de la Sociedad de Biología de Concepción (Chile) 59: 95-104

JEREZ V (1991) El género Dyctyneis Baly, 1865 (Coleoptera: Eumolpinae). Taxonomía, distribución geográfica y descripción de nueva especie. Gayana Zoología (Chile) 55: 31-52

JEREZ V (1992) Revisión taxonómica del género Procalus Clark, 1865 (Chrysomelidae: Alticinae). Gayana Zoología (Chile) 56: 109-126

JEREZ V (1995) Stenomela pallida Erichson, 1847. Redescripción, ontogenia y afinidad con el género Hornius (Chrysomelidae-Eumolpinae). Gayana Zoología (Chile) 1: 1-12

JEREZ V (1996) Biology and phylogenetic remarks of the sub-antarctic genera Hornius, Stenomela and Dictyneis (Chrysomelidae: Eumolpinae). En: Jolivet PHA \& ML Cox (eds) Chrysomelidae Biology: 239258. Volume 3. General Studies. SPB Academic Publishing, Ámsterdam, The Netherlands.

JEREZ V (2000a) Diversidad y patrones de distribución geográfica de insectos coleópteros en ecosistemas desérticos de la Región de Antofagasta, Chile. Revista Chilena de Historia Natural 73: 79-92.

JEREZ V (2000b) Microestructura coriónica en huevos de Lysathia atrocyanea (Phil. \& Phil.) (Coleoptera: Chrysomelidae). Revista Chilena de Entomología 27: 71-73

JEREZ V (2005) Patrones de diversidad de Chrysomelidae (Insecta- Coleóptera) en la Cordillera de la Costa de Chile central y sur. En: Smith-Ramírez C, JJ Armesto y CC. Valdovinos (eds) Historia, biodiversidad y ecología de los bosques costeros de Chile: 340-351. Editorial Universitaria, Santiago, Chile. 708 pp

JEREZ V \& H IBARRA-VIDAL (1992) Morphology and Bionomics of Hornius grandis (Phil. \& Phil, 1864) (Chrysomelidae, Eumolpinae). Boletín de la Sociedad de Biología de Concepción (Chile) 63: 93 100.

JEREZ V \& C CENTELLA (1996) Primer registro de nemátodos Mermithidae, parásitos de Procalus mutans y Procalus reduplicatus (Chrysomelidae: Alticinae). Acta Entomológica Chilena 20: 107-110.

KUSCHEL G (1957) La subfamilia Aterpinae en América (Ap. 12 de Coleoptera Curculionidae). Revista Chilena de Entomología 1: 205-245.

KUSCHEL G (2000) La fauna curculiónica (Coleoptera:
Curculionidae) de la Araucaria araucana. Revista Chilena de Entomología 27: 41-51.

LÓPEZ E \& M GUTIÉRREZ (1983) Listado de nombres geográficos. Tomo I y II. Instituto Geográfico Militar, Santiago, Chile. 1.557 pp.

MENDOZA G \& V JEREZ (2001) Coleópteros epígeos asociados a Peumus boldus Mol. en la Reserva Nacional Isla Mocha, Chile. Gayana Zoología (Chile) 65: 129-136.

MOORE T (1981) Aporte al conocimiento de bupréstidos en Chile (Coleoptera: Buprestidae). Revista Chilena de Entomología 11: 37-68.

MOORE T (1985) Aporte al conocimiento de los bupréstidos de Chile (Coleoptera: Buprestidae). Segunda nota. Revista Chilena de Entomología 12: 113-139.

MOORE T (1986) Aporte al conocimiento de los bupréstidos de Chile (Coleoptera: Buprestidae). Tercera contribución. Revista Chilena de Entomología 13: 37-46.

MOORE T (1987) Aporte al conocimiento de los bupréstidos de Chile (Coleóptera: Buprestidae). Cuarta contribución. Revista Chilena de Entomología 15: 13-19.

MOORE T (1990) Aporte al conocimiento de los bupréstidos de Chile (Coleoptera: Buprestidae). Quinta contribución. Revista Chilena de Entomología 18: 89-91.

MORRONE JJ (1993) Revisión sistemática de un nuevo género de Rhytirrhinini (Coleoptera: Curculionidae), con un análisis biogeográfico del dominio Subantártico. Boletín de la Sociedad de Biología de Concepción (Chile) 64: 121-145.

MORRONE JJ (1994) On the identification of areas of endemism. Systematic Biology 43: 438-441.

MORRONE JJ (2004) Panbiogeografia, componentes bióticos y zonas de transición. Revista Brasileira de Entomología 48: 149-162.

MORRONE JJ \& A RUGGIERO (2001) Como planificar un análisis biogeográfico. Dugesiana 7: 1-8.

MYERS N, R MITTERMEIER, C MITTERMEIER, G FONSECA \& J KENT (2000) Biodiversity hotspots for conservation priorities. Nature 403: 853-858.

NEWTON A \& M THAYER (2003) Austral species of Staphylinidae, Silphidae, Leiodidae and Agyrtidae (online). Field Museum of Natural History, Chicago, Illinois, USA; http:// www.fieldmuseum.org/peet_staph/db_1c.html.

NIXON KC (2000) Winclada Ver. 0.9.99.55.mexu (Beta). Published by the author, Ithaca, New York, USA.

NUMHAUSER J (1985) Revisión del género Pycnosiphorus grupo I Lessoni Weinneich (Coleópteros: Lucanidae). Revista Chilena de Entomología 12: 53-75.

O'BRIEN CH (1971) The biogeography of Chile through entomofaunal regions. Entomological News 82: 197-207.

OVALLE M \& J SOLERVICENS (1980) Observaciones sobre la biología de Megathopa villosa Eschscholtz, 1822 (Coleoptera, Scarabaeidae; Scarabaeinae). Boletín del Museo Nacional de Historia Natural (Chile) 37: 235-246.

PACE R (2000) Aleocharinae del Cile, nuove o poco note (Coleoptera, Staphylinidae). Bolletino del Museo Regionale di Scienze Naturali di Torino (Italia) 17: 355-510.

PEÑA L (1971) Revisión del género Nycterinus Eschscholtz, 1829 (Coleoptera-Tenebrionidae). Boletín del Museo Nacional de Historia Natural (Chile) 32: 129-158. 
PEÑA L (1996) Introducción al estudio de los insectos de Chile. Cuarta edición. Editorial Universitaria, Santiago, Chile. 253 pp.

PIZARRO-ARAYA J \& V JEREZ (2004) Distribución geográfica del género Gyriosomus, GuérinMéneville, 1834 (Coleoptera, Tenebrionidae): una aproximación biogeográfica. Revista Chilena de Historia Natural 77: 491-500.

PLISCOFF P (2003) Priorización de áreas para fortalecer la conservación de la flora arbórea nativa en la zona mediterránea de Chile. Tesis de Magister, Facultad de Ciencias, Universidad de Chile, Santiago, Chile. $102 \mathrm{pp}$; (Online) http://www.cybertesis.cl/tesis/ uchile/2002/pliscoff_p

POLYMERIS C (1995) Vegetación actual de la Península de Hualpén. Clasificación y dinámica. Tesis de Magister, Facultad de Ciencias Naturales y Oceanográficas, Universidad de Concepción, Concepción, Chile. 190 pp.

POSADAS P \& D MIRANDA-ESQUIVEL (1999) El PAE (Parsimony analysis of endemicity) como una herramienta en la evaluación de la biodiversidad. Revista Chilena de Historia Natural 72: 539-546.

RAW A (2004) Leafcutter and mason bees: a biological catalogue of the genus Megachile of the Neotropics (online). Departamento de Ciencias Biológicas, Universidad Estatal de Santa Cruz, Brasil. 97 pp.; http://www.webbee.org.br/raw

ROIG-JUÑENT S (1994) Las especies chilenas de Cnemalobus Guérin-Meneville 1838 (Coleoptera: Carabidae: Cnemalobini). Revista Chilena de Entomología 21: 5-30.

ROIG-JUÑENT S, J CRISCI, P POSADAS \& S LAGOS (2002) Áreas de distribución y endemismos en zonas continentales. En: Costa C, SA Vanin, JM Lobo \& A Melic (eds) Proyecto de Red Iberoamericana de Biogeografía y Entomológica Sistemática: 247-266. Monografías del Tercer Milenio, Volumen 2. Sociedad Entomológica Aragonesa (SEA), Zaragoza, España.

ROIG-JUÑENT S \& G DEBANDI (2004) Prioridades de conservación aplicando información filogenética y endemicidad: un ejemplo basado en Carabidae (Coleoptera) de América del Sur austral. Revista Chilena de Historia Natural 77: 695-709.

ROVITO S, MTK ARROYO \& P PLISCOFF (2004) Distributional modelling and parsimony analysis of endemicity of Senecio in the Mediterranean-type climate area of central Chile. Journal of Biogeography 31: 1623-1636.

SALGADO JM (1991) Nuevos datos sobre Cholevidae y Camiaridae (Coleoptera) de Chile. Elytron 5: 169-179.

SCOTT JM \& MD JENNINGS (1998) Large-area mapping of biodiversity. Annals of the Missouri Botanical Garden 85: 34-47

SIMONETTI J (2000) Diversidad Biológica. En: Hoffman A (ed) Informe País. Estado del Medio Ambiente en Chile-1999: 175-194. Centro de Análisis de Políticas Públicas, Universidad de Chile, Santiago, Chile.

SOLERVICENS J (1986) Revisión taxonómica del género Eurymetopum Blanchard (Coleoptera, Cleridae, Phyllobaeninae). Acta Entomológica Chilena 13: 11-120.
SOLERVICENS J (1987) Silviella, nuevo género de Phyllobaeninae (Coleoptera: Cleridae) de la parte meridional de América del sur. Acta Entomológica Chilena 14: 25-40.

SOLERVICENS J (1991) Revisión del género Eurytomorphon Pic, 1950 (Coleoptera, Cleridae, Clerinae). Acta Entomológica Chilena 16: 20-36.

SOLERVICENS J (1995) Consideraciones generales sobre los insectos, el estado del conocimiento y las colecciones. En: Simonetti J, M Arroyo, A Spotorno \& E Losada (eds) Diversidad biológica de Chile: 198-210. Comisión Nacional de Ciencia y Tecnología, Santiago, Chile.

SOLERVICENS J (1996) Revisión taxonómica del género Notocymatodera Schenkling, 1907 (Coleoptera: Cleridae). Acta Entomológica Chilena 20: 99-106.

SOLERVICENS J (2001) Revisión taxonómica del género Solervicensia Barr, 1979 (Coleoptera: Cleridae: Korynetinae). Acta Entomológica Chilena 25: 77 84.

SOLERVICENS J (2002) Catálogo de los cléridos de Chile (Coleoptera: Cleridae). Acta Entomológica Chilena 26: 81-94.

SOLERVICENS J (2005) Diversidad y endemismo de Cleridae (Coleoptera) del Parque Nacional Nahuelbuta. En: Smith-Ramírez C, JJ Armesto \& C Valdovinos (eds) Historia, biodiversidad y ecología de los bosques costeros de Chile: 324-339. Editorial Universitaria, Santiago, Chile.

SOLERVICENS J \& P ESTRADA (2002) Insectos epígeos de asociaciones vegetacionales esclerófilas de la Reserva Nacional de Río Clarillo (Región Metropolitana, Chile). Acta Entomológica Chilena 26: 27-44.

TENEB EA, L CAVIERES, $M$ PARRA \& A MARTICORENA (2004) Patrones geográficos de distribución de árboles y arbustos en la zona de transición climática mediterráneo-templada de Chile. Revista Chilena de Historia Natural 77: 5171.

VELOSO A \& H NÚÑEZ (1998) Inventario de fauna de la Región de Antofagasta: recursos metodológicos e información. Revista Chilena de Historia Natural 71: 555-569.

VILLAGRÁN C \& L HINOJOSA (1997a) Historia de los bosques del sur de Sudamérica I: antecedentes paleobotánicos, geológicos y climáticos del Terciario del cono sur de América. Revista Chilena de Historia Natural 70: 225-239.

VILLAGRÁN C \& L HINOJOSA (1997b) Historia de los bosques del sur de Sudamérica II: Análisis fitogeográfico. Revista Chilena de Historia Natural 70: 241-267.

VILLAGRÁN C \& JJ ARMESTO (2005) Fitogeografía histórica de la Cordillera de la Costa de Chile. En: Smith-Ramírez C, J Armesto y C Valdovinos (eds) Historia, biodiversidad y ecología de los bosques costeros de Chile: 99-116. Editorial Universitaria, Santiago, Chile.

YEATES DK, P BOUCHARD \& GB MONTEITH (2002) Patterns and levels of endemism in the Australian wet tropics rainforest: evidence from flightless insects. Invertebrate Systematics 16: 605-619. 